\title{
A Bayesian Approach for Analysis of Whole-Genome Bisulfite Sequencing Data Identifies Disease-Associated Changes in DNA Methylation
}

\author{
Owen J. L. Rackham, ${ }^{*, 1}$ Sarah R. Langley, ${ }^{*, 1}$ Thomas Oates, ${ }^{+, 1}$ Eleni Vradi, ${ }^{\ddagger 1}$ \\ Nathan Harmston,* Prashant K. Srivastava, Jacques Behmoaras, ** Petros Dellaportas, ${ }^{\dagger t, \neq \neq, 2}$ \\ Leonardo Bottolo, ${ }^{\neq \neq, \S \S, * * *, 2}$ and Enrico Petretto*,t,2 \\ *Duke-National University of Singapore Medical School, 169857, Singapore, ${ }^{\dagger}$ Medical Research Council, London Institute of \\ Medical Sciences, Imperial College London, W12 ONN, §Division of Brain Sciences, Faculty of Medicine, and ${ }^{* *}$ Centre for \\ Complement and Inflammation Research, Imperial College London, SW7 2AZ, United Kingdom, ${ }^{\ddagger}$ Department of Statistics, Athens \\ University of Economics and Business, GR10434, Greece, ${ }^{\dagger}{ }^{\dagger}$ Department of Statistical Science, University College London, SW7 2AZ, \\ United Kingdom, ${ }^{\ddagger \neq}$ The Alan Turing Institute, London, NW1 2QR, United Kingdom, $\$ \S$ Department of Medical Genetics, University \\ of Cambridge, CB2 0QQ, United Kingdom, and ${ }^{* * *}$ Medical Research Council Biostatistics Unit, Cambridge Institute of Public \\ Health, CB2 OSR, United Kingdom
}

\begin{abstract}
DNA methylation is a key epigenetic modification involved in gene regulation whose contribution to disease susceptibility remains to be fully understood. Here, we present a novel Bayesian smoothing approach (called ABBA) to detect differentially methylated regions (DMRs) from whole-genome bisulfite sequencing (WGBS). We also show how this approach can be leveraged to identify disease-associated changes in DNA methylation, suggesting mechanisms through which these alterations might affect disease. From a data modeling perspective, ABBA has the distinctive feature of automatically adapting to different correlation structures in CpG methylation levels across the genome while taking into account the distance between $\mathrm{CpG}$ sites as a covariate. Our simulation study shows that ABBA has greater power to detect DMRs than existing methods, providing an accurate identification of DMRs in the large majority of simulated cases. To empirically demonstrate the method's efficacy in generating biological hypotheses, we performed WGBS of primary macrophages derived from an experimental rat system of glomerulonephritis and used ABBA to identify > 1000 disease-associated DMRs. Investigation of these DMRs revealed differential DNA methylation localized to a 600 bp region in the promoter of the Ifitm3 gene. This was confirmed by ChIP-seq and RNA-seq analyses, showing differential transcription factor binding at the Ifitm3 promoter by JunD (an established determinant of glomerulonephritis), and a consistent change in Ifitm3 expression. Our ABBA analysis allowed us to propose a new role for Ifitm3 in the pathogenesis of glomerulonephritis via a mechanism involving promoter hypermethylation that is associated with Ifitm3 repression in the rat strain susceptible to glomerulonephritis.
\end{abstract}

KEYWORDS Bayesian statistics; DNA methylation; WGBS; glomerulonephritis

NE of the most important epigenetic modifications directly affecting DNA is methylation, where a methyl group is added to a cytosine base in the DNA sequence creating 5-methylcytosine. High-throughput sequencing techniques, such as whole-genome bisulfite sequencing (WGBS), now allow for genome-wide methylome data to be collected at single-base resolution (Harris et al. 2010). However, the challenge remains how to accurately identify DNA methylation changes at the genome-wide level, and also account for the complex correlation structures present in the data. While it is still not fully understood how DNA methylation affects gene expression, it has been shown that, depending on the location of the modification, it can either have a positive or negative effect on the level of expression of genes (Gutierrez-Arcelus et al. 2013). How methylation patterns are regulated is complex, and a full understanding of this process requires elucidating the mechanisms for de novo DNA methylation and demethylation, as well as the maintenance of methylation (Chen and Riggs 2011). However, the majority of functional methylation changes are found in methylation sites where cytosines are immediately followed by guanines, known as CpG dinucleotides (Ziller et al. 2011). These are not positioned randomly across the genome, but tend to appear in clusters called CpG islands (CpGI) (Deaton and Bird 2011). It has been also shown that there are concordant methylation changes within CpGI, and in the genomic regions immediately 
surrounding CpGI (also known as CpGI shores or CpGS). These "spatially correlated" DNA methylation patterns tend to be more strongly associated with gene expression changes than the methylation changes occurring in other parts of the genome (Gutierrez-Arcelus et al. 2015). The correlation of methylation levels between CpG sites is also highly dependent on their genomic context, varying greatly depending on where in the genome they are located (Zhang et al. 2015). For computational convenience, the dependence of methylation patterns between CpG sites is sometimes ignored by methods for differential methylation analysis. Alternatively, a simplified estimation of the correlation of methylation levels between neighboring CpG sites (Bell et al. 2011) based on a user-defined parameterization of the degree of smoothing is introduced. These strategies might not be appropriate across different experimental scenarios, and, instead, we propose an automatic probabilistic smoothing procedure of the average methylation levels across replicates (hereafter methylation profiles).

Beyond the initial univariate analysis of methylation changes at each individual CpG (for instance, using the Fisher's exact test), the focus has shifted recently to identifying differentially methylated regions (DMRs), since coordinated changes in CpG methylation across genomic regions are known to impart the strongest regulatory influence. With this aim, a number of tools have been proposed to detect DMRs from WGBS data. Typically, these methods normally take one of two approaches: either model the number of methylated/unmethylated reads using a binomial, negative-binomial distribution or discrete distributions with an overdispersion parameter) such as MethylKit (Akalin et al. 2012), MethylSig (Park et al. 2014), and DSS (Feng et al. 2014). Alternatively, in order to account for the correlation of methylation profiles between neighboring CpG sites, a smoothing operator is applied in tools like BSmooth (Hansen et al. 2012), BiSeq (Hebestreit et al. 2013), DSS-single (Wu et al. 2015) - reviewed in Robinson et al. (2014) and Yu and Sun (2016b). Methods based on spline- (Hansen et al. 2012), and kernel- (Hebestreit et al. 2013) generally perform well in practical applications. However, their results, and the identification of the DMRs depend on the choice of the smoothing parameters values, e.g., window size or kernel bandwidth, a feature that makes them less general, and prone to perform unequally when the default parameters

Copyright @ 2017 Rackham et al.

doi: https://doi.org/10.1534/genetics.116.195008

Manuscript received August 19, 2016; accepted for publication February 3, 2017; published Early Online February 16, 2017.

Available freely online through the author-supported open access option.

This is an open-access article distributed under the terms of the Creative Commons Attribution 4.0 International License (http://creativecommons.org/licenses/by/4.0/), which permits unrestricted use, distribution, and reproduction in any medium, provided the original work is properly cited.

Supplemental material is available online at www.genetics.org/lookup/suppl/doi:10 1534/genetics.116.195008/-/DC1.

${ }^{1}$ These authors contributed equally to this work

${ }^{2}$ Corresponding authors: Department of Statistical Science, University College, Gower

Street, London WC1E 6BT, UK. E-mail: p.dellaportas@ucl.ac.uk; Department of Medical Genetics, University of Cambridge, Cambridge Biomedical Campus, CB2 0QQ, UK.

E-mail: Ib664@cam.ac.uk; and Duke-NUS Medical School, 8 College Road, Singapore

169857, Republic of Singapore. E-mail: enrico.petretto@duke-nus.edu.sg values are changed. In these cases, smoothing parameters tuned by time-consuming sensitivity analysis based on different parameterizations is usually recommended, although this strategy is rarely applied in real data analyses. Other approaches, e.g., metilene (Jühling et al. 2015), propose segmentation algorithms to detect DMRs between single/groups of replicates without making any model assumption about the data generating mechanism, and are less dependent on parameter definition. Furthermore, several other algorithms have been introduced, e.g., MOABS (Sun et al. 2014), Lux (Äijö et al. 2016), and MACAU (Lea et al. 2015), showing that bisulfite sequencing data analysis is an active area of research.

To address this dependence on parameterization, and the subsequent lack of generality, we propose a fully Bayesian approach: approximate Bayesian bisulfite sequencing analysis (ABBA). ABBA is designed to smooth automatically the underlying - not directly observable-methylation profiles and reliably identify DMRs while borrowing information vertically across biological replicates and horizontally across correlated CpGs (Figure 1). We highlight that this fully Bayesian specification is not adopted by previous DMR detection techniques, owing to the computational overhead of the inferential procedure. We address the high computational demands by utilizing a highly efficient inferential tool (Rue et al. 2009) for Bayesian models (see below, and Materials and Methods). To demonstrate the benefits of adopting ABBA over existing approaches, we report a comprehensive simulation study, where we benchmarked ABBA against five commonly used alternative methods (Fisher Exact Test, BSmooth, MethylKit, MethylSig, and DSS), considered a proposed new one (metilene), and assessed the effect of a different biological and experimental conditions (by varying parameters related to data integrity and quality of the signal) on the performance of each method. The results from this benchmark clearly indicate that ABBA is the best performing method, being both robust to changes in factors affecting data quality (e.g., sequencing coverage and errors associated with the methylation call), and level of noise in methylation signal. To benchmark our proposed method on a real dataset, we generated new WGBS data in macrophages from an established rat model of glomerulonephritis (Aitman et al. 2006) and control strain, and used ABBA for the genomewide identification of DMRs. An additional comparison performed with the best alternative method (arising from the simulation study) showed that ABBA has increased power to detect changes in DNA methylation involving genes and pathways relevant to glomerulonephritis. Furthermore, this comparison exemplifies how the DMR results obtained by alternative approaches depend heavily on the choice of relevant smoothing parameters (e.g., window size used in DSS). We also integrated the DMR results of ABBA with transcription factor binding site analysis, RNA-seq and ChIP-seq data generated in the same system, and, in this, we revealed a previously unappreciated role for the Ifitm 3 gene in the pathogenesis of glomerulonephritis, providing a proof-of-concept for real data applications of the ABBA approach. 


\section{Materials and Methods}

Below, we report the key aspects of the latent Gaussian model and Integrated Nested Laplace Approximation (INLA). The interested reader can also refer to Rue and Martino (2007) and Rue et al. (2009).

\section{Latent Gaussian model}

A latent Gaussian model (LGM) can be described by a threestage hierarchical model

$$
\begin{aligned}
y_{i} \mid x_{i}, \boldsymbol{\theta} & \sim \pi\left(y_{i} \mid x_{i}, \boldsymbol{\theta}\right), \\
\boldsymbol{x} \mid \boldsymbol{\theta} & \sim N\left(\boldsymbol{\mu}(\boldsymbol{\theta}), \boldsymbol{Q}^{-1}(\boldsymbol{\theta})\right), \\
\boldsymbol{\theta} & \sim \pi(\boldsymbol{\theta}),
\end{aligned}
$$

where $y_{i}, i=1, \ldots, n$, are the observed values, $\boldsymbol{x}$ is an $n$ dimensional vector of latent variables, and $\boldsymbol{\theta}$ is $p$-dimensional vector of model parameters. (1) is the observations equation, and describes the probabilistic model for each observation conditionally on the latent variable $x_{i}$ and the model parameters $\boldsymbol{\theta}$; (2) is the latent Gaussian field equation, with the latent variables distributed as a $p$-dimensional normal distribution, with mean vector $\boldsymbol{\mu}(\boldsymbol{\theta})$, and a sparse precision matrix $\boldsymbol{Q}(\boldsymbol{\theta})$. Both quantities can depend on the model parameters vector, $\boldsymbol{\theta}$, whose distribution is described in the parameter equation (3). The Gaussian vector, $\boldsymbol{x}$, exhibits a particular conditional dependence (or Markov) structure which is reflected in its precision matrix $\boldsymbol{Q}(\boldsymbol{\theta})$.

\section{Integrated nested Laplace approximation}

INLA is a computational approach to perform statistical inference for LGM. It provides a fast and accurate alternative to exact Markov chain Monte Carlo (MCMC) (Gilks et al. 1996), and other sampling-based methods such as Sequential Monte Carlo (SMC) (Doucet et al. 2001). They become prohibitively computationally expensive when the length of the sequence considered is too long, resulting in infeasible run times. The INLA solution, with a mix of Laplace approximations (Tierney and Kadane 1986) and numerical integrations offers a pragmatic inferential tool to fit LGMs, and it provides answers in hours, whereas MCMC requires days. The INLA inferential procedure consists of three steps:

1. Compute the approximation to the marginal posterior $\pi(\boldsymbol{\theta} \mid \boldsymbol{y})$ and byproduct to $\pi\left(\theta_{j} \mid \boldsymbol{y}\right), j=1, \ldots, p$;

2. Compute the approximation to $\pi\left(x_{i} \mid \boldsymbol{y}, \boldsymbol{\theta}\right), i=1, \ldots, n$;

3. Combine 1 and 2 above, and compute the approximation to the marginal posterior $\pi\left(x_{i} \mid \mathbf{y}\right)$.

\section{ABBA model}

Based on LGM, the ABBA model can be described by a threestage hierarchical model:

$$
y_{i g r} \mid \pi_{i g r} \sim \operatorname{Binomial}\left(n_{i g r}, \pi_{i g r}\right),
$$

$$
\begin{gathered}
\operatorname{logit}\left(\pi_{i g r}\right) \mid \sigma_{g}^{2} \sim \mathrm{N}\left(\mu_{i g}, \sigma_{g}^{2}\right) \\
\mu_{i g} \mid \rho_{i g}^{2} \sim \mathrm{N}\left(\mu_{i-1, g}, \rho_{i g}^{2}\right) \\
\sigma_{g}^{-2} \sim \operatorname{Gam}(0.1,0.1) \\
\rho_{i g}^{2}=\rho_{g}^{2}\left|p_{i}-p_{i-1}\right| \text { with } \rho_{g}^{-2} \sim \operatorname{Gam}(0.1,0.1)
\end{gathered}
$$

(4) is the first part of the observations equation, where $i=1, \ldots, m$ denotes the $\mathrm{CpG}, g=1,2$ the group (e.g., case and control group), and $r=1, \ldots, R$ the experimental replicate. $y_{i g r}, n_{i g r}$, and $\pi_{i g r}$ are the observed number of methylated reads, the read depth, and the proportion of methylation for the ith CpG site, gth group, and the experimental replicate, respectively. (5) is the second part of the observations equation, and it describes a random effect across the experimental replicates, with a specific variance $\sigma_{g}^{2}$ for each group. In (5), $\operatorname{logit}(z)$ indicates the logit transformation, $\operatorname{logit}(z)=\log [1 /(1-z)]$. The observation Equation (5) assumes that the methylation proportions are drawn from the same distribution within each group, but are different between groups.

Equation (6) is the latent Gaussian field (LGF) equation. The dependence of the DNA methylation pattern between CpGs is modeled as a nonstationary random walk of order 1 , RW(1): $\mu_{i g}$ follows a normal distribution, with mean $\mu_{i-1, g}$ [defined in the $(i-1)$ th $\mathrm{CpG}$ ], and variance $\rho_{i g}^{2}$, which is specific for each $\mathrm{CpG}$ and group. Equations (5) and (6) highlight an important feature of the ABBA model that it is able to model vertically the information contained in the replicates by a random effect model and horizontally the information about the CpG methylation levels correlation by a LGF.

The model is completed by specification in (7) and (8) of the random effect and LGM prior precision, i.e., the inverse of the variance. For computational convenience, we introduce a $\mathrm{CpG}$ site spacing and decompose $\rho_{i g}^{2}$ into $\rho_{g}^{2}\left|p_{i}-p_{i-1}\right|$, where $\rho_{g}^{2}$ is the global smoothing parameter specific for each group that needs to be estimated, and $p_{i}$ and $p_{i-1}$ are the chromosomal locations of two consecutive $\mathrm{CpG}$ sites. This implies that the correlation between $\mu_{i g}$ and $\mu_{i-1, g}$ depends on the distance between the two consecutive CpG sites, and, in particular, it decreases as this distance increases, in keeping with empirical evidence reported in Bell et al. (2011), Zhang et al. (2015), and in our real data set (see Supplemental Material, Figure S1). With this formulation, only $\sigma_{g}^{2}$ and $\rho_{g}^{2}$ need to be estimated for each group. It also implies a sparse precision matrix $\boldsymbol{Q}(\boldsymbol{\theta})$ for the LGF in (2), making the overall inferential process efficient.

Finally, noninformative priors are assigned to the precision parameters $\sigma_{g}^{-2}$ and $\rho_{g}^{-2}$, which are distributed as a gamma density with mean 1 and variance 10 (default INLA values). Sensitivity analysis on the gamma density parameterization shows no departure from the results obtained using the 
A

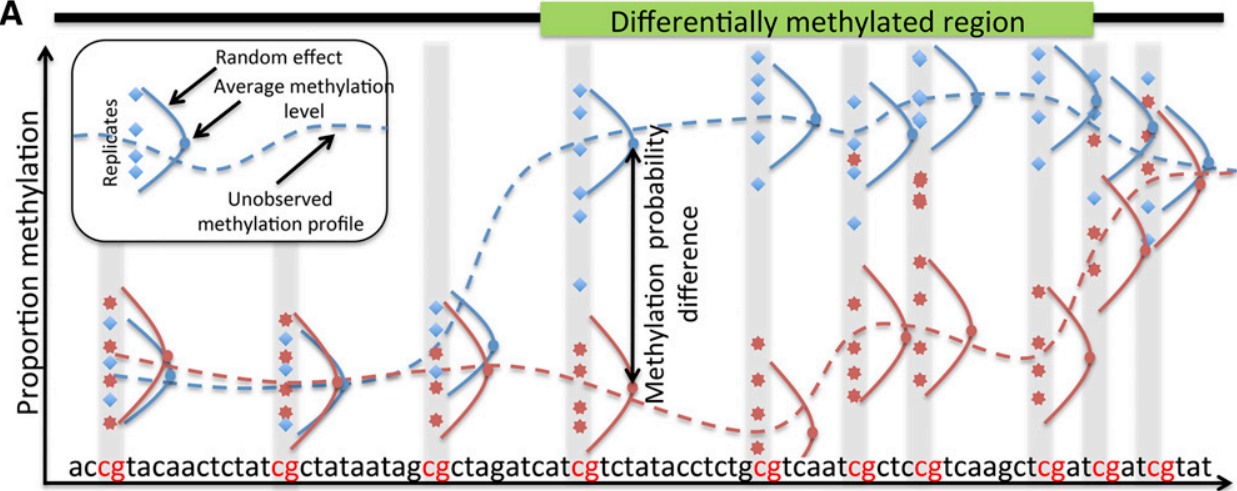

B

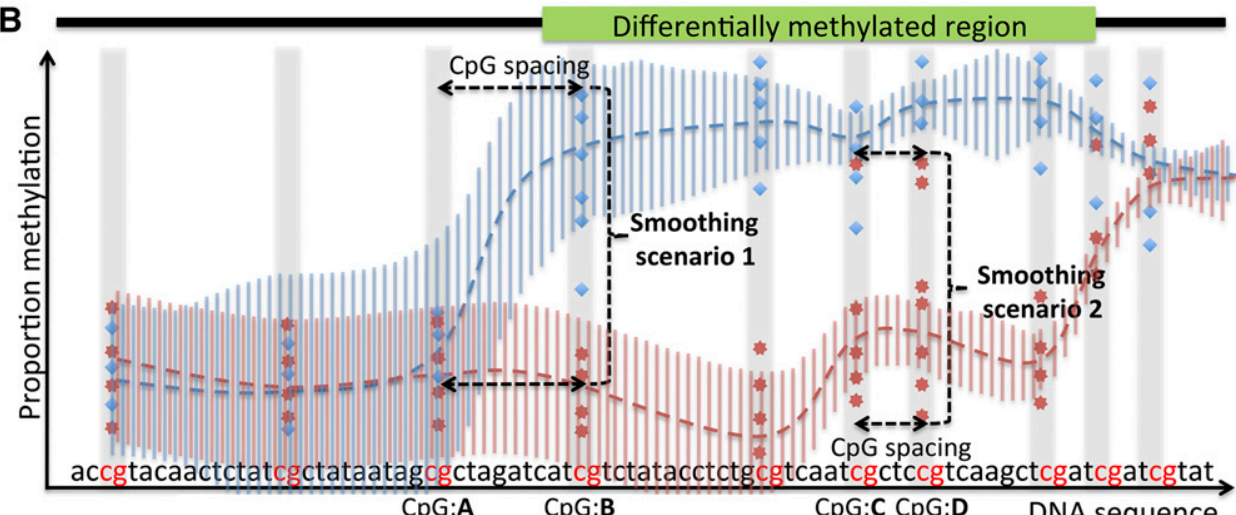

Figure 1 ABBA model. ABBA estimates the unobserved methylation profiles, i.e., the DNA average methylation levels across replicates, of two groups from WGBS data (blue diamonds and red stars). (A) A random effect accounts for the variability of experimental replicates. At each $\mathrm{CpG}$, the methylation probability difference is the difference between the methylation profile of the two groups (blue and red dots). (B) The methylation profiles of each group are smoothed by a latent Gaussian field that probabilistically connects them (dotted lines). In particular "Smoothing scenario 1" shows that if a large spacing (distance) between two consecutive $\mathrm{CpGs}$ (CpG:A and CpG: B) exists, the methylation profile at $\mathrm{CpG}: \mathrm{B}$ does not depend on the previous one at CpG:A (blue dotted line). The opposite happens in "Smoothing scenario 2" where the methylation profile at CpG:D is largely influenced by the previous one at CpG:C (red dotted line) despite some high levels of methylation (red stars), which are treated by ABBA as outliers. The degree of the smoothing, i.e., the correlation between DNA methylation profiles, is controlled automatically by the marginal variance of the Latent Gaussian Field (blue and red vertical bars): the cor-

relation is higher (lower) when the variance is small (large). On the other hand, the variance decreases as the distance between neighboring CpGs' decreases (Smoothing scenario 2) while it increases as the distance increases (Smoothing scenario 1).

default values. See Table S1 for details on the posterior density of $\sigma_{g}^{-2}$ and $\rho_{g}^{-2}$ under INLA default and alternative parameterization on selected simulated examples.

When a single replicate is available, since $\sigma_{g}^{2}=0,(4)$ and (5) simplify to

$$
\begin{gathered}
y_{i g} \mid \pi_{i g} \sim \operatorname{Binomial}\left(n_{i g}, \pi_{i g}\right), \\
\operatorname{logit}\left(\pi_{i g}\right)=\mu_{i g} .
\end{gathered}
$$

While some methods for DMR detection (Feng et al. 2014; Wu et al. 2015), allow for overdispersion by assuming a beta-binomial model, (4) and (5) imply a logistic-normal model. After integrating out (6), $\quad \int \mathrm{N}\left(\operatorname{logit}\left(\pi_{i g r}\right) \mid \mu_{i g}, \sigma_{g}^{2}\right) \mathrm{N}\left(\mu_{i g} \mid \mu_{i-1, g}, \rho_{i g}^{2}\right) d \mu_{i g}=$ $\mathrm{N}\left(\operatorname{logit}\left(\pi_{i g r}\right) \mid \mu_{i-1, g}, \sigma_{g}^{2}+\rho_{i g}^{2}\right)$, it can be shown that marginally

$$
\begin{aligned}
\mathrm{V}\left(Y_{i g} \mid \sigma_{g}^{2}, \rho_{i g}^{2}\right) \approx & n_{i g} \pi_{i g}\left(1-\pi_{i g}\right) \\
& \times\left\{1+\left(\sigma_{g}^{2}+\rho_{i g}^{2}\right)\left(n_{i g}-1\right) \pi_{i g}\left(1-\pi_{i g}\right)\right\},
\end{aligned}
$$

where $\pi_{i g} \equiv \exp \left(\mu_{i g}\right) /\left[1+\exp \left(\mu_{i g}\right)\right]$. The above equation illustrates that, a priori, the marginal degree of variability per CpG site under the ABBA model is the variance of the binomial model multiplied by an overdispersion factor that depends on the combined effect of $\sigma_{g}^{2}$, the replicates variability, and $\rho_{i g}^{2}$, the variance of the unobserved methylation profile. When a single replicate is available, the overdispersion depends only on $\rho_{i g}^{2}$.

\section{ABBA algorithm}

The ABBA algorithm consists of two steps:

1. Compute the approximation to the marginal posteriors of $\sigma_{g}^{2}$, the variance of the random effect, and $\rho_{g}^{2}, g=1,2$ the smoothing parameters; given the model specification $\rho_{i g}^{2}=\rho_{g}^{2}\left|p_{i}-p_{i-1}\right|$, it is also possible to derive the marginal posteriors of $\rho_{i g}^{2}$;

2. Compute the approximation to marginal posterior $\pi\left(\mu_{i g} \mid \mathbf{y}\right)$, where $\boldsymbol{y}=\left(y_{i g r}\right)_{i=1, \ldots, n: g=1,2: r=1, \ldots . R}$; then the marginal posterior of the unobserved methylation profile $\pi\left(\pi_{i g} \mid \boldsymbol{y}\right)$ is obtained by using the inverse logit transformation of $\mu_{i g}$, $z \equiv \exp \{\operatorname{logit}(z)\} /[1+\exp \{\operatorname{logit}(z)\}]$.

\section{Global differential methylation and false discovery rate (FDR) calculation}

ABBA inference about DMRs is based on the posterior methylation probability (PMP) $\pi\left(\boldsymbol{\pi}_{i g} \mid \boldsymbol{y}\right)$ and the posterior differential methylation probability (PDMP) $\pi\left(\pi_{i 1} \mid y\right)-\pi\left(\pi_{i 2} \mid y\right)$. The posterior mean methylation probability $\mathrm{E}\left(\boldsymbol{\pi}_{i g} \mid \boldsymbol{y}\right)$ summarizes 

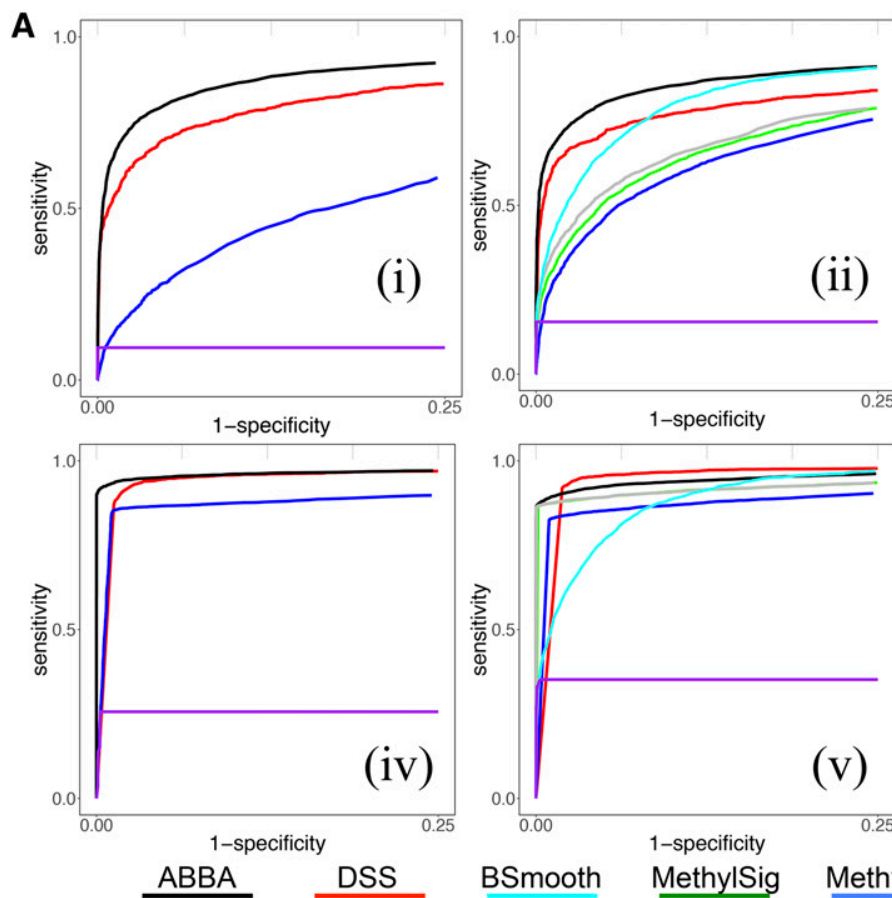

(ii)
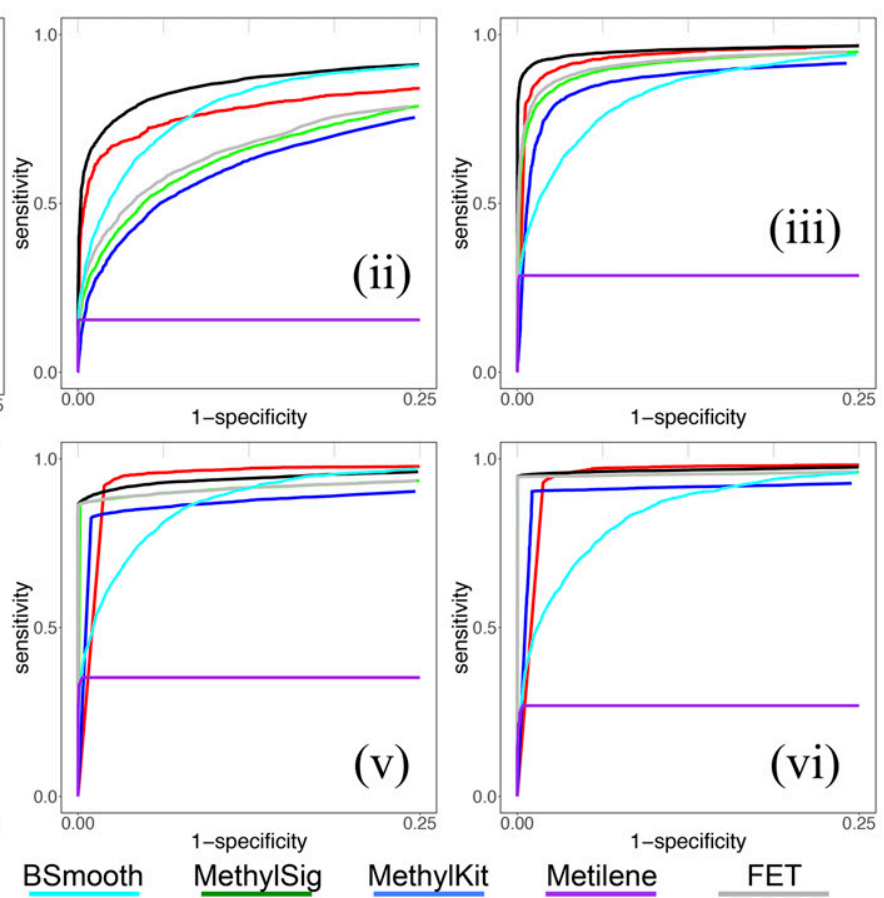

B

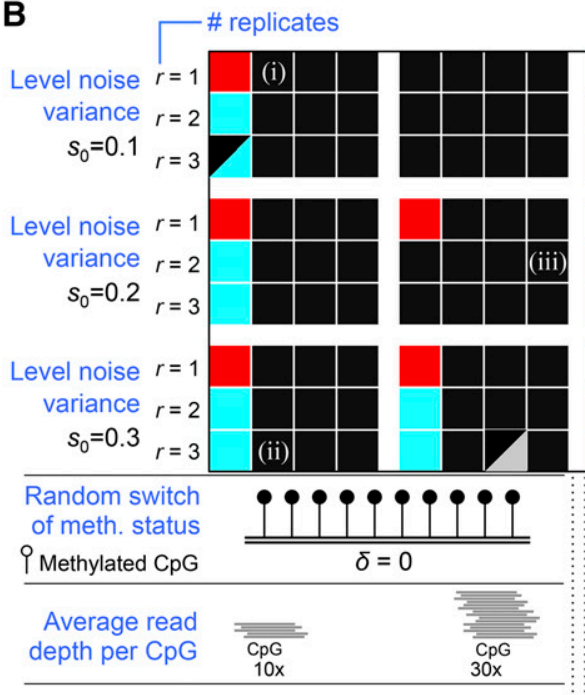

Ameth (\%) $20 \quad 305070 \quad 20305070$
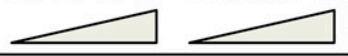

C

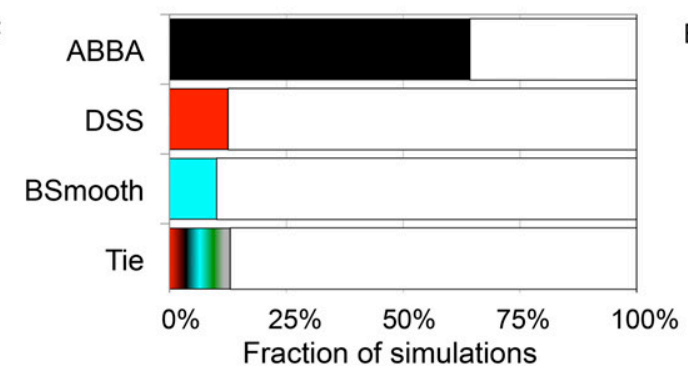

Benchmark grid

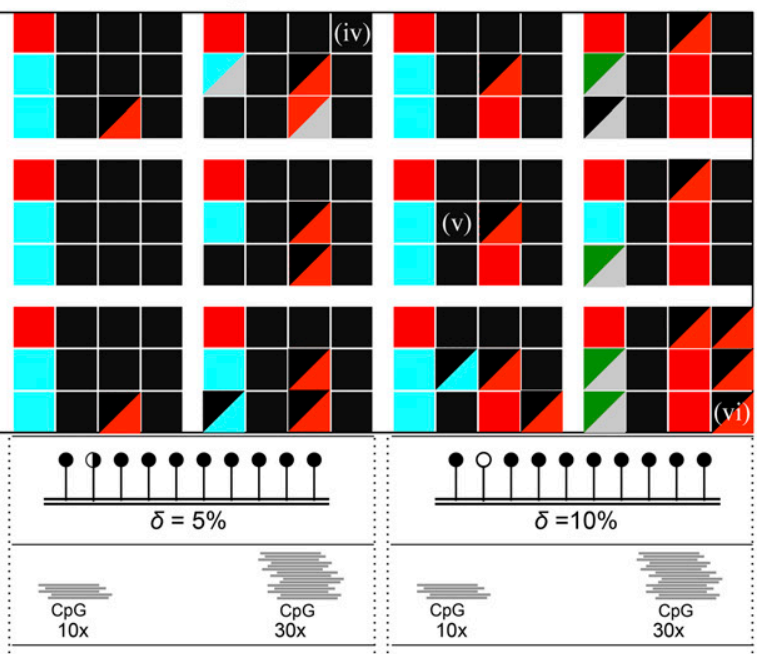

$20305070 \quad 20305070 \quad 20305070 \quad 20305070$

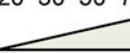

Figure 2 Benchmarking results (A) ROC curves for selected combinations of parameters: (i) $s_{0}=0.1$, $\Delta$ meth $=30 \%, \quad r=1$, average read depth per CpG of $10 \times$, $\delta=0$; (ii) $s_{0}=0.3, \Delta$ meth $=$ $30 \%, r=3$, average read depth per CpG of $10 \times, \delta=0$; (iii) $s_{0}=0.2, \Delta$ meth $=70 \%, r=2$, average read depth per $\mathrm{CpG}$ of $30 \times$, $\delta=0$; (iv) $s_{0}=0.1, \Delta$ meth $=$ $70 \%, r=1$, average read depth per CpG of $30 \times, \delta=5 \%$; (v) $s_{0}=0.2, \Delta$ meth $=30 \%, r=2$, average read depth per $\mathrm{CpG}$ of $10 \times$, $\delta=10 \%$; (vi) $s_{0}=0.3, \Delta$ meth $=$ $70 \%, r=3$, average read depth per CpG of $30 \times, \delta=10 \%$. For each of this combination of parameters, the corresponding best method based on its pAUC is indicated in the benchmark grid below. In (i) and (iv), ROC curves are reported only for the methods that can analyze WGBS data generated from one biological sample. (B) Global snapshot of the method's performance across 216 simulated datasets. A given combination of parameters is indicated by a square in the benchmark grid, and, for each square, we calculated the pAUC for each method and determined which method had the overall best pAUC (i.e., pAUC method_1 $>$

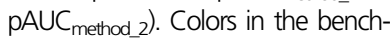
mark grid indicate which method had the best performance. When pAUC of two methods are similar $( \pm 1 \%)$ we report the colors of both methods (e.g., black and red colors in the same square indicate similar performance of $A B B A$ and DSS). The six selected combination of parameters for which the ROC curves are reported in $(A)$ are indicated within the benchmark grid: (i-vi). All ROC curves are reported in Figure S5, Figure S6, and Figure S7. (C) For the three best performing methods (ABBA, DSS, and $B S m o o t h)$, we report the percentage of simulated scenarios in which each method resulted to be the best based on the pAUC comparison. "Tie" indicates the proportion of simulated scenarios in which the pAUCs of any two methods were similar (i.e., pAUCs $\pm 1 \%$ ), and it was not possible to single out a single best performing approach. the information contained in the PMP, and is used to define the posterior mean differential methylation between two groups, $d_{i}=\mathrm{E}\left(\pi_{i 1} \mid \boldsymbol{y}\right)-\mathrm{E}\left(\pi_{i 2} \mid \boldsymbol{y}\right)$. Once the LGF has been integrated out by INLA inferential process, $\pi\left(\pi_{i g} \mid \boldsymbol{y}\right), i=1, \ldots, n$, and, in turn $d_{i} \mathrm{~s}$, become marginally independent. This allows the straightforward application of a nonparametric FDR procedure without 
A

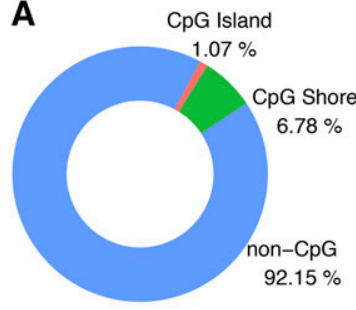

B

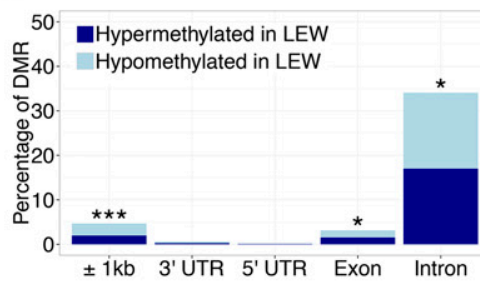

C

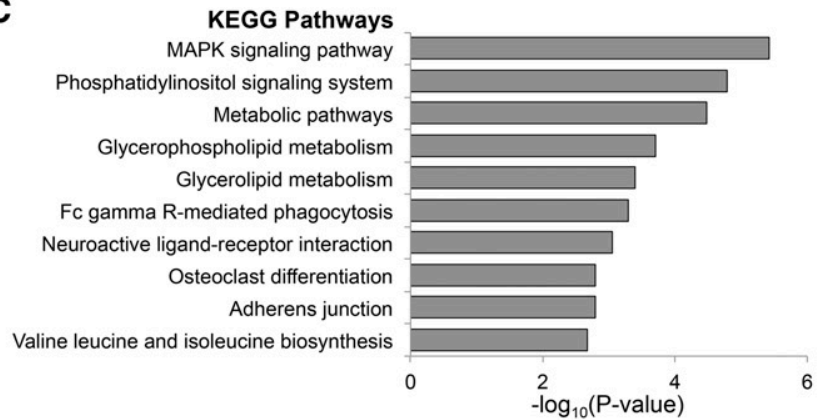

D

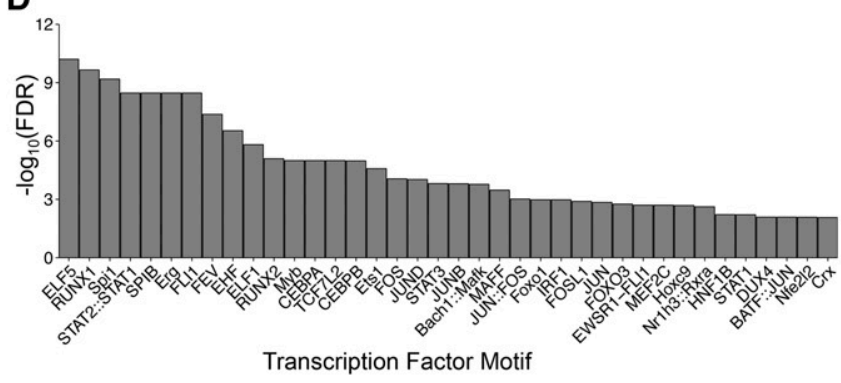

E

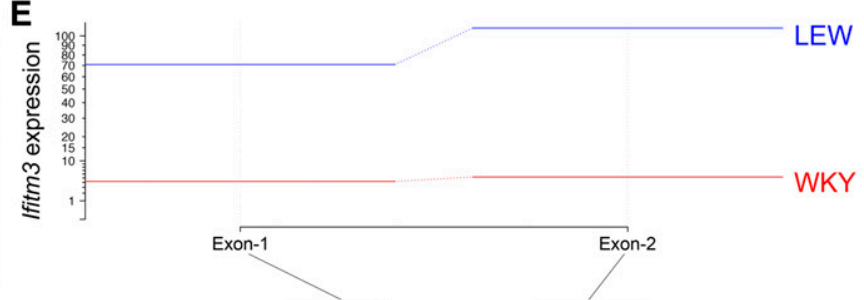

WKY
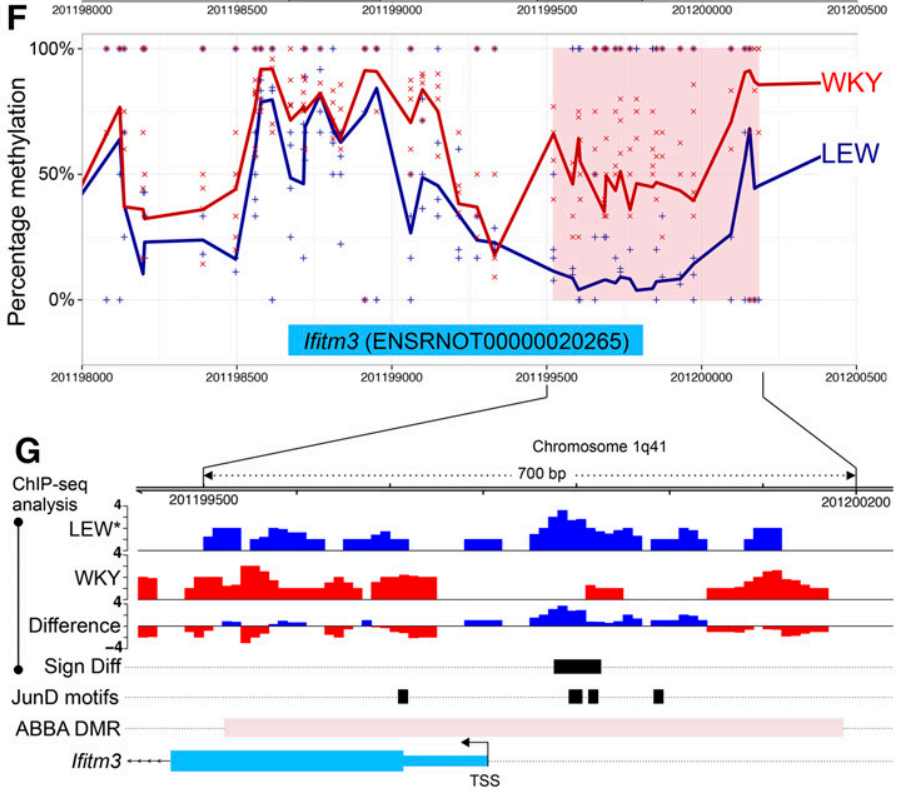

LEW*, WKY.LCrgn2

Figure 3 ABBA analysis of WGBS in rat macrophages. (A) CpG-based annotation 1004 DMR between WKY and LEW macrophages showing significantly higher proportions of $\mathrm{CpGl}$ and $\mathrm{CpGS}$ than those that would be expected by chance $(P$-value $<0.009$ for $C p G l$, and $P$-value $<0.001$ for $C p G S$, respectively, obtained by 1000 randomly sampled datasets of 1004 CpG-matched regions). (B) Proportions of DMRs in different genomic features of overlapping genes. Feature annotation was retrieved from UCSC genome browser (RN4). (C) KEGG pathway enrichment for the genes overlapping with DMRs. Only significant pathways are reported (FDR $<1 \%$ ). (D) Enrichment for the TFBS within the DMRs was when compared to CG matched regions of the genome (FDR < 0.05). (E) RNA-seq analysis in WKY and LEW macrophages shows lack of Ifitm3 expression in WKY rats. (F) Percentage methylation at each CpG in WKY (crosses) and LEW (plus), and smoothed average methylation profiles by ABBA. The pink box highlights the significant DMR identified by ABBA (FDR < 5\%). (G) ChIP-seq analysis for JunD in LEW.LCrgn2 (LEW*), and WKY macrophages identified a single region with differential binding of JunD ( $P$-value $<0.05$, Sign Diff row, black box). Units on the $y$-axis refer to relative ChIP-seq coverage with respect to the control. This region overlapped with two (out of four) JunD binding sites motifs identified within the gene promoter ( $\pm 500 \mathrm{bp}$ around the TSS). ABBA DMR, differentially methylated region identified by ABBA. TSS, transcription start site. * $P$-value $<0.05, * * * P$-value $<0.001$.

the burden of correlated signals. To distinguish between the null distribution (no differential methylation) and the alternatives, we fit a mixture of three truncated normal densities

$$
\begin{aligned}
d_{i} \sim & \pi_{-} \mathrm{N}_{[-1,1]}\left(\theta_{-}, \xi_{-}^{2}\right)+\pi_{0} \mathrm{~N}_{[-1,1]}\left(\theta_{0}, \xi_{0}^{2}\right) \\
& +\pi_{+} \mathrm{N}_{[-1,1]}\left(\theta_{+}, \xi_{+}^{2}\right),
\end{aligned}
$$

where $\mathrm{N}_{[-1,1]}$ is a normal density truncated between $[-1,1]$, $\pi_{-}, \pi_{0}, \pi_{+} \in(0,1)$ with $\pi_{-}+\pi_{0}+\pi_{+}=1$ are the mixing weights of the "negative" differentially methylated, no differentially methylated, and "positive" differentially methylated with respect the control group, respectively, $\theta_{-}, \theta_{0}, \theta_{+}$are the unknown centers of the differentially methylated groups, and $\xi_{-}^{2}, \xi_{0}^{2}, \xi_{+}^{2}$ are the unknown variances. Under the null hypothesis, we set $\theta_{0}=0$. For identifying the components of mixture model, we also impose the condition $\pi_{0} \geq \pi_{-}+\pi_{+}$, under the assumption that the large majority of $\mathrm{CpG}$ sites are not differentially methylated.

Although the choice of a three component mixture model works well in real data examples (see Figure S2), this assumption can be relaxed. For instance, as suggested in Sun and Cai (2009), the non-null distribution $f_{1}$ can have more than two components. This allows a better fitting of the tails of distribution of $d_{i}$ s and the identification of more than two differentially methylated groups. For instance the choice of the number of components can be based on Bayesian Information Criterion (BIC). However, this requires running the FDR procedure several times for each choice of the number of components. Another possibility that is less computationally intensive relies on the approximation of $f_{1}$ by using a nonparametric Gaussian kernel density estimation (Kuan and Chiang 2012). 
Maximum likelihood estimates of (9) are obtained by the EM algorithm (Dempster et al. 1977), taking particular care to avoid local maxima in the likelihood surface by running the EM algorithm from different starting points. Using the EM algorithm, the posterior probability of a CpG site belonging to each of the three components is

$$
\begin{gathered}
\mathrm{P}\left(z_{i}=“-"\right)=\frac{\pi_{-} \mathrm{N}_{[-1,1]}\left(d_{i} ; \theta_{-}, \xi_{-}^{2}\right)}{C}, \\
\mathrm{P}\left(z_{i}=“ 0 "\right)=\frac{\pi_{0} \mathrm{~N}_{[-1,1]}\left(d_{i} ; 0, \xi_{0}^{2}\right)}{C}, \\
\mathrm{P}\left(z_{i}=“+"\right)=\frac{\pi_{+} \mathrm{N}_{[-1,1]}\left(d_{i} ; \theta_{+}, \xi_{+}^{2}\right)}{C}
\end{gathered}
$$

with

$C=\pi_{-} \mathrm{N}_{[-1,1]}\left(d_{i} ; \theta_{-}, \xi_{-}^{2}\right)+\pi_{0} \mathrm{~N}_{[-1,1]}\left(d_{i} ; 0, \xi_{0}^{2}\right)+\pi_{+} \mathrm{N}_{[-1,1]}$ $\left(d_{i} ; \theta_{+}, \xi_{+}^{2}\right)$.

Similarly to Broët et al. (2004), for a constant $t$, we define the estimated $\operatorname{FDR}(t)$ as

$$
\widehat{\operatorname{FDR}}(t)=\frac{\sum_{i \in \mathcal{I}_{-}} \mathrm{P}\left(z_{i}=“ 0 ”\right)+\sum_{i \in \mathcal{I}_{+}} \mathrm{P}\left(z_{i}=“ 0 ”\right)}{n_{-}+n_{+}}
$$

where $\mathcal{I}_{-}=\left\{i: d_{i} \leq-t\right\}, \mathcal{I}_{+}=\left\{i: d_{i} \geq t\right\}, n_{-}=\#\left(\mathcal{I}_{-}\right)$ and $n_{+}=\#\left(\mathcal{I}_{+}\right)$. Equation (10) defines the global FDR as the average local FDR which, for posterior probabilities, is defined as $1-\mathrm{P}\left(z_{i}="-"\right)-\mathrm{P}\left(z_{i}="+"\right)=\mathrm{P}\left(z_{i}=" 0 "\right)$. Finally, the constant $t$ is chosen such that FDR $(t) \leq$ FDR.

In summary, the FDR procedure for ABBA consists of two steps:

1. Fit a mixture of truncated normal densities with three components on the $d_{i}$ s values; obtain the posterior probability that each $d_{i}$ belongs to each of the three components;

2. Calculate the constant $t$, such that FDR $(t) \leq$ FDR for a desired level of FDR.

For computational efficiency, our FDR procedure can be run on each chromosome separately and then the results can be aggregated at the genome-wide level (Efron 2008). Besides the computational speed, this strategy does not assume the existence of a global methylation level difference between the two conditions that may not hold in practice. The separate-class model (Efron 2008), can be used to combine separate chromosome-wide FDRs.

\section{WGBS data simulation}

WGBS data have a number of intrinsic characteristics that can vary depending on the cell-types/tissue complexity being studied, or on technical issues related to the sequencing. In order to assess which method is the most robust for analyzing WGBS data, it is important that changes in each of these characteristics are taken into account. Here, we take advantage of our previously published WGBS-data simulator
(Rackham et al. 2015) that allows us to generate unbiased benchmarking datasets with several varying parameters. Wherever possible, we will refer to the notation used in Rackham et al. (2015); the parameters are the following:

1. Number of replicates-the parameter $r$ was set to vary between $r=1,2,3$ within each group;

2. Average read depth-at each CpG site for all replicates and groups, the number of reads $n_{i g r}, i=1, \cdots, m$ and $g=1,2$, is simulated using a Poisson distribution with average read depth $\lambda$. The parameter $\lambda$ was set to be either 10 or 30 reads on average per CpG site;

3. Level of noise - the parameter $s_{0}$ controls the level of noise added the probability of methylation at each $\mathrm{CpG}$ site for all replicates and groups, and simulates the measurement error resulting from the sampling of DNA segments during sequencing $\pi_{i r g}=\operatorname{logit}{ }^{-1}\left(\operatorname{logit}\left(\pi_{r g}\right)+\varepsilon_{i}\right)$, where $\pi_{r g}$ is the global probability of methylation of the binomial (emission) distribution based on the real dataset analyzed [see details in Rackham et al. (2015)], and $\varepsilon_{i} \sim \mathrm{N}\left(0, s_{0}\right)$, $i=1, \ldots, m$. $s_{0}$ was set to vary between $0.1,0.2$ and 0.3 to model different level of noise. To calibrate the value of $s_{0}$, Table S2 provides a Monte Carlo estimation of the effect of different values of the noise level on $\pi_{i r g}$.

4. Methylation probability difference-the parameter $\Delta$ meth reported in Rackham et al. (2015) as "phase difference" controls the magnitude of the difference between the probabilities of methylation in each group, and was set to vary between $20,30,50$, and $70 \%$. This difference is obtained on CpG sites where both case and control samples share the same methylated status (methylated or unmethylated), by adding a given value to the probability in either cases or controls. The total length of the sequence where this difference appears in no greater than 5\% (WGBSSuite default value) of the total length of the simulated region.

5 . We also considered an additional parameter $\delta$ (not available for modeling in WGBSSuite), which introduces a further error associated with the methylation call. After selecting at random with a given probability $\delta$ a CpG site in the gth group for all replicates, we switch its methylation status between the two groups. In our simulation study, the parameter $\delta$ has been varied from 0 , to 0.05 , to 0.1 .

To perform the benchmarking, we generated five replicates of $5000 \mathrm{CpGs}$ for each combination of the above parameters. The resulted in a total of 216 benchmarking datasets (three cases for the number of replicates, two cases for the average read depth, three cases for the level of noise, four cases for the methylation probability difference, and three cases for the parameter $\delta$ ), which are replicated five times $(5,400,000$ CpGs in total) to assess the Monte Carlo average performance for each combination of parameters. In these datasets, the size of the differentially methylated regions has a median size of 15 CpGs (see Figure S3). The proportion of differentially methylated CpGs cannot exceed $20 \%$ of all CpGs (i.e., $~ 1000$ CpGs). 


\section{Receiver operator curve (ROC) construction for benchmarking}

In order to generate the ROC curve, the performance is calculated CpG-wise. For a given DMR, detection of each of the CpG contained within is considered as a true positive, while $\mathrm{CpGs}$ that are not detected are considered false negatives. Outside of the DMR, the opposite criteria is applied. We choose this assignment criteria rather than calling detection of a each DMR since it provides a useful quantification of the extent each DMR is captured by each technique; for instance, if one technique correctly identifies all the CpGs in a DMR, the method is deemed to perform better than an approach that identifies correctly only $80 \%$ of the CpGs within the same DMR.

\section{WGBS data preprocessing for ABBA}

To run ABBA efficiently at the genome-wide level, we took advantage of a cluster-computing environment that enables parallel computation, and with this aim we preprocessed the WGBS data as follows. After the raw WGBS data were aligned, we removed $\mathrm{CpG}$ sites where $<50 \%$ of the samples contain reads. Next, we split the WGBS data into chunks such that the distance between the last CpG site in one chunk and the first CpG in the next chunk is $>3000$ bp. It has been previously shown that the correlation of DNA methylation levels between CpG sites decreases dramatically after $400 \mathrm{bp}$ (Zhang et al. 2015), so splitting the data in this way implies a particular conditional dependence structure in our data defined by a sparse block-diagonal precision matrix $\boldsymbol{Q}(\boldsymbol{\theta})$, where each block corresponds to a WGBS chunk. Chunks were then analyzed in parallel in a cluster-computing environment. We calculated the time required by ABBA to analyze chunks of different length (that span from $100 \mathrm{CpGs}$ to $15,000 \mathrm{CpGs}$ ) on a single machine, with $202.3-\mathrm{GHz}$ hyper-threaded cores and 32 GB of RAM, and found that the computational time (seconds) scales with the chunk length ( $N_{\mathrm{CpG}}$, number of CpG sites) following the power function: time (seconds) $=0.0045 N_{\mathrm{CpG}}{ }^{1.3985}\left(R^{2}=0.997\right)$. Depending on the genome length and data dimensionality, a complete WGBS analysis ABBA might require days (e.g., it took $\sim 2$ weeks to analyze WGBS data in the rat). The total computational time of ABBA analysis can be significantly shortened by splitting the genome into smaller chunks, and then assembling the result. The results provided by the "whole-genome" ABBA analysis, and "smaller-chunks" ABBA analyses are highly consistent, with no differences in the distribution probabilities obtained with and without splitting the genome into chunks (Figure S4). Scripts for the preprocessing step are embedded within ABBA at http://abba.systemsgenetics.net/.

\section{WGBS of rat macrophages}

Bone-marrow derived macrophages (BMDM) were isolated from WKY and LEW rat strains. WGBS libraries were produced as follows: $6 \mu \mathrm{g}$ of genomic DNA was spiked with $10 \mathrm{ng}$ of unmethylated cl857 Sam7 lambda DNA (Promega, Madison, WI), and sheared using a Covaris System S-series model S2. Sheared DNA was purified, and then end-repaired in a $100 \mu \mathrm{l}$ reaction using NEBNext End Repair kit (New England Biolabs, Beverly, MA) incubated at $20^{\circ}$ for $30 \mathrm{~min}$. Endrepaired DNA was next A-tailed using NEBNext dA-tailing reaction buffer and Klenow Fragment (also New England Biolabs) incubated at $37^{\circ}$ for $30 \mathrm{~min}$, and then purified with the MinElute PCR purification kit (Qiagen) in a total final elution volume of $28 \mu$ l. Illumina Early Access Methylation adapter oligos (Illumina) were then ligated to a total of $25 \mu \mathrm{l}$ of the A-tailed DNA sample using NEBNext Quick Ligation Reaction Buffer and Quick T4 DNA ligase (both New England Biolabs) in a reaction volume of $50 \mu$ l. This mixture was incubated for $30 \mathrm{~min}$ at $20^{\circ}$ prior to gel purification. Bisulfite conversion of $450 \mathrm{ng}$ of the purified DNA library was achieved using the Epitect Bisulfite kit (Qiagen) in a total volume of $140 \mu \mathrm{l}$. Samples were incubated with the following program: $95^{\circ}$ for $5 \mathrm{~min}, 60^{\circ}$ for $25 \mathrm{~min}, 95^{\circ}$ for $5 \mathrm{~min}$, $60^{\circ}$ for $85 \mathrm{~min}, 95^{\circ}$ for $5 \mathrm{~min}$, and $60^{\circ}$ for $175 \mathrm{~min}$, and then $3 \times$ repeat of $95^{\circ}$ for $5 \mathrm{~min}$ and $60^{\circ}$ for $180 \mathrm{~min}$ and held at $20^{\circ}$. Treated samples were then purified as per the manufacturer's instructions. Adapter bound DNA fragments were amplified by a 10-cycle PCR reaction and then purified using Agencourt AMPure XP beads (Beckman Coulter) before gel extraction and quantification using the Agilent Bioanalyzer 2100 Expert High Sensitivity DNA Assay. Then, libraries were quantified using quantitative PCR, then denatured into single stranded fragments. These fragments were then amplified by the Illumina cluster robot, and transferred to the HiSequation 2000 for sequencing. WGBS reads were aligned and filtered according to a previously published pipeline (see Johnson et al. 2012, 2014). Briefly, reads were preprocessed by in silico conversion of $\mathrm{C}$ bases to $\mathrm{T}$ bases in read 1 , and $\mathrm{G}$ bases to A bases in read 2, followed by clipping of the first base from each read. Preprocessed reads were aligned to the rat reference genome (RGSC3.4) using BWA version 0.6.1 (Li and Durbin 2009), with $3^{\prime}$ end quality trimming using a $Q$ score cutoff of 20. Converted and clipped reads 1 and 2 were mapped to two in silico converted versions of the reference sequence, first with Cs converted to Ts to allow forward strand mapping, and second with Gs converted to As to allow mapping of reverse strand. Aligned reads were filtered by removal of clonal reads, reads with a mapping quality of $<20$, reads that mapped to both in silico converted forward and reverse strands, and reads with an invalid mapping orientation. We obtained 79.9 billion "mappable" bases across both rat strains, with $13.5 \times$ (average) coverage in the Lew strain and $17.6 \times$ (average) in WKY, where the greatest depth of coverage was observed within CpG islands.

Despite ABBA being able to detect methylation changes at all genomic locations, we focused only on those methylation changes that occur at $\mathrm{CpG}$ sites, and considered $\mathrm{CpG}$ sites where at least four out of the eight samples contain reads (resulting in a total of 14,976,632 CpG sites genome-wide in BMDM from WKY and LEW rats). DMRs were called with 
ABBA (see above) using a 5 CpG minimum, a 33\% or greater difference in methylation, and a 5\% FDR threshold. Genomic region annotations and Ensembl gene IDs for the rat reference genome 4 (rn4), were downloaded from the UCSC genome browser. Significant over-representations of genomic features (intron, exons, etc.) were determined empirically from 1000 randomly sampled length, and GC-matched regions per DMR. The genes overlapping with DMRs were further annotated and tested for enrichment in Kyoto Encyclopedia of Genes and Genomes (KEGG) pathways using WebGestalt (Wang et al. 2013).

Identification of enriched transcription factor binding site (TFBS) motifs within the DMRs identified by ABBA was performed using HOMER (Heinz et al. 2010). HOMER was used to scan for motifs obtained from the JASPAR 2014 database (Mathelier et al. 2014). Threshold used for motif identification was a $P$-value of $10^{-4}$. Enrichments were calculated by comparing the motifs present in the DMRs against a large set of background sequences $\left(N=10^{6}\right)$ corrected for $\mathrm{CpG}$ content.

\section{RNA-seq and ChIP-seq analysis of rat macrophages}

RNA-seq data from BMDM in WKY and LEW strains were retrieved from Rotival et al. (2015), and reanalyzed in the context of WGBS analysis reported here. Briefly, total RNA was extracted from BMDM at day 5 of differentiation in three WKY rats and three LEW rats using Trizol (Invitrogen). Total RNA ( $1 \mu \mathrm{g}$ ) was used to generate RNA-seq libraries using TruSeq RNA sample preparation kit (Illumina, Little Chesterford, UK). Libraries were run on a single lane per sample of the HiSequation 2000 platform (Illumina) to generate $100 \mathrm{bp}$ paired-end reads. An average depth of $72 \mathrm{M}$ reads per sample was achieved (minimum $38 \mathrm{M}$ ). RNA-seq reads were aligned to the rn4 reference genome using tophat2. The average number of mapped was $67 \mathrm{M}$ (minimum $36 \mathrm{M}$ ) corresponding to an average mapping percentage of $93 \%$. Sequencing and mapping were quality controlled using the FastQC software. Gene-level read counts were computed using HT-Seq-count (Anders et al. 2015) with "union" mode, and genes with $<10$ aligned reads across all samples were discarded prior to analysis leading to 15,155 genes. Differential gene expression analysis between WKY and LEW BMDMs was performed using DESeq2 (Love et al. 2014), and significantly differentially expressed genes were reported at the 5\% FDR level. Visualizations of the expression levels with gene structure were created with DEXSeq (Anders et al. 2012).

ChIP-seq data from BMDM isolated from the WKY and WKY.LCrgn2 congenic strains (in which the LEW Crgn2 QTL was introgressed onto the WKY background) were retrieved from Hull et al. (2013) and Srivastava et al. (2013) and reanalyzed with respect to the Ifitm3 locus. This congenic model (WKY.LCrgn2) has been extensively studied in previous studies, where it has been shown that JunD expression levels are significantly higher in WKY when compared with the congenic (Hull et al. 2013), and that the canonical binding of
AP-1 is significantly greater in WKY compared to WKY. LCrgn2 (Behmoaras et al. 2008). Briefly, ChIP was performed with a JunD antibody (Santa Cruz sc74-X), and a negative IgG control (sc-2026). Single read library preparation, and high throughput single-read sequencing for 36 cycles was carried out on an Illumina Genome Analyzer IIx and sequencing of the ChIP-seq libraries was carried out on the high throughput Illumina Genome Analyzer II. Initial data processing was performed using Illumina Real Time Analysis (RTA) v1.6.32 software (equivalent to Illumina Consensus Assessment of Sequence and Variation, CASAVA 1.6) using default settings. Quality filtered reads were then realigned to the rn4 using the Burrows Wheeler Alignment tool v0.5.9 (BWA). Read ends were trimmed if Phred-scaled base quality scores dropped to $<20$. For the ChIP-seq analysis presented in Figure 3G, differences in JunD binding were assessed only within a $700 \mathrm{bp}$ region spanning the Ifitm 3 gene promoter, which included the 600 bp-long DMR identified by ABBA at this locus. ChIP-seq differences were assessed by means of Fisher's exact test on the ChIP-seq counts (normalized for library size) in WKY LCrgn2 and LEW strains, respectively, using a sliding window of $50 \mathrm{bp}$. This locus-specific analysis identified a single 50 bp window with differential JunD binding with FET $P$-value $<0.05$ that overlapped with JunD TFBS motifs identified by HOMER (see above).

\section{Data availability}

ABBA is implemented as a Perl/R program, which is available with instructions for download at http://abba.systemsgenetics.net/ or via http://www.mrc-bsu.cam.ac.uk/software/ bioinformatics-and-statistical-genomics/. The data are available on Gene Expression Omnibus (GEO), https://www.ncbi.nlm. nih.gov/geo/, under the accession number GSE84719.

\section{Results}

We employ a fully Bayesian approach (a Bayesian structured generalized mixed additive model with a latent Gaussian field), which models the random sampling process of the WGBS experiment (the number of methylated/unmethylated reads distributed as non-Gaussian response variable), and where all the unknown quantities are specified by probability distributions. To perform inference, ABBA takes advantage of INLA (Rue et al. 2009), a new inferential tool for latent Gaussian models. INLA provides approximations to the posterior distribution of the unknowns. These approximations are both very accurate and extremely fast to compute compared to established exact sampling-based methods such as MCMC (Gilks et al. 1996) or SMC (Doucet et al. 2001). Our new proposed algorithm ABBA is therefore the combination of an approximate inferential procedure with a fully Bayesian model tailored for bisulfite sequencing analysis.

ABBA calculates the PMP at each CpG site based on an estimate of the posterior probability of a smoothed unobserved methylation profile. It also identifies DMRs at a specified FDR by contrasting PMPs across the whole-genome 
between two groups, e.g., cases and controls. Several intrinsic features of WGBS data are incorporated into ABBA: for instance, the variability in DNA methylation between the (experimental) replicates within each group is modeled through a random effect with a specific within-group variance (Figure 1A). The correlation of DNA methylation patterns is encoded in the latent Gaussian field equation, which reflects the neighborhood structure of the model, and automatically adapts to the changes in the underlying data. In particular, the a priori correlation between neighboring CpGs' methylation profiles depends on the distance between them, as it decreases as this distance increases (Figure 1B). Rather than relying on a user-defined value to parameterize it (e.g., kernel bandwidth or window size), or fixing it by an automatic procedure (for instance through an empirical Bayes approach), ABBA assigns a prior distribution to the parameters of the latent Gaussian field equation, thus fully accounting for the uncertainty about these quantities. This specification is key in our model, since the data-adaptivity of the degree of smoothing conforms better to the data than assuming fixed values. All of these features allow our model to adjust routinely to real-world scenarios, providing an automatic way to describe the WGBS data without requiring any user-defined parameters (Yu and Sun 2016b). Full technical details of the ABBA algorithm can be found in the Materials and Methods section.

We benchmarked ABBA and compared it against recently proposed methods [MethylKit (Akalin et al. 2012), MethylSig (Park et al. 2014), DSS/DSS-single (Feng et al. 2014; Wu et al. 2015), simply DSS hereafter, BSmooth (Hansen et al. 2012), metilene (Jühling et al. 2015), and the univariate Fisher's exact test (FET)]. All methods were run using their default parameterization, and, for the FET, we pooled data from different replicates. To ensure a fair comparison, we used WGBSSuite (Rackham et al. 2015) to generate a large number of diverse datasets that were independent of the underlying statistical models of ABBA and of the other methods. Briefly, we simulated in silico datasets to assess the performance of each method under several scenarios, which reflect differences in data integrity, and the quality of the signal that can occur as a result of biological and experimental phenomena. The parameters considered were the following: the number of replicates within each group $(r)$, the average read depth per $\mathrm{CpG}$, the level of noise variance $\left(s_{0}\right)$, the methylation probability difference between the two groups ( $\Delta$ meth), and the switching of methylation status of CpG sites between the two groups $(\delta)$ (see Materials and Methods for details). For each simulated case, we generated five replicates, and compared the accuracy of the CpGs called as being contained within DMRs by each technique with the true simulated DMRs. To quantitatively assess the performance of ABBA with respect to competing methods, we evaluated falsepositive and false-negative rates of $\mathrm{CpG}$ sites, and generated ROC curves. We focused on the partial area under the ROC curve (or pAUC) at a specificity of 0.75 . The pAUC is considered to be more practically relevant than the area under the entire ROC curve (Ma et al. 2013), since, in typical genomics studies, only the features identified at very low false positive rates are selected for further biological validation.

All results of the benchmark are detailed in Figure S5, Figure S6, and Figure S7. Figure 2A shows representative ROC curves from a specific combination of parameters, while in Figure 2B we summarize the performance over all combinations of parameters by displaying the best performing method based on its pAUC. Specifically, in Figure 2B, the color code in the "benchmark grid" indicates the best performing method for each of the 216 simulated scenarios. For instance, in Figure 2A, the top left panel (i) shows the ROC curves for all methods considered under a simulated dataset with $s_{0}=0.1, \Delta$ meth $=30 \%, r=1$, average read depth per CpG of $10 \times$, and $\delta=0$. For this combination of parameters, we compared the pAUC of each approach, which shows that ABBA is the best performing method. Accordingly, in Figure 2B, the square in the grid that represents this parameter set [indicated by (i) in the figure] is colored black (ABBA). Examples of other ROC curves for specific combinations of parameters are reported in Figure 2A, i-vi, and the corresponding best performing methods are indicated in Figure 2B. In some simulated cases (e.g., with high levels of $\delta=10 \%$ ) the ROC curves, and corresponding pAUC, do not distinguish unambiguously the best performing method (e.g., Figure 2A, vi). In these cases when the pAUC of two methods are very similar $( \pm 1 \%)$, we report the colors of both methods, e.g., black and red colors in the same square to indicate similar performance of ABBA and DSS (Figure 2B). For the metilene approach (Jühling et al. 2015) (which was run using its default parametrization), we noticed that ROC curve analysis was not suitable to compare its performance with other methods. Specifically, for metilene, we found that it was not possible to assess both specificity and sensitivity across the wide range of DMRs and scenarios simulated in our study. Representative examples for the ROC curves obtained by running metilene (and other approaches) on the simulated data are provided in Figure 2A and in Figure S8.

Considering all 216 simulated datasets, and comparing the pAUCs obtained by each approach across all combinations of parameters, ABBA (black) proved to be the best performing method in 139 (64\%) cases (Figure 2, B and C). The two other competitive methods were DSS and BSmooth, which were the best performing approach only in 26 (12\%) and 22 (10\%) simulated cases, respectively (Figure 2, B and C). In 28 (13\%) cases, different methods showed very similar performance (i.e., pAUCs $\pm 1 \%$ ), and, in 17 simulations, ABBA and DSS showed comparable performance. Looking at the detailed ROC curves reported in Figure S5, Figure S6, and Figure S7, we notice that, while ABBA was the best method across all simulations (Figure 2C), its performance diminished for simulated datasets, with a very small methylation probability difference between the two groups. In particular, for most of the simulated scenarios with $\Delta$ meth $=20 \%$, BSmooth showed very good and robust performance, while DSS was consistently the best performing method when $r=1$ and $\Delta$ meth $=20 \%$, Figure 2B. However, we 
highlight that such small differences in DNA methylation (i.e., $\Delta$ meth $\leq 20 \%$ ) are unlikely to have an important biological effect, and the most commonly observed effect sizes for DMR range between 20 and $40 \%$, as previously reported (Ziller et al. 2015). In the range $\Delta$ meth $\geq 30 \%$, ABBA was the best performing method in $132(81 \%)$ simulations, while DSS was the best performing method only in 10 (6\%) simulated cases, and, notably, BSmooth was never the best single performing method (BSmooth showed similar performance of ABBA in only one simulated case) (Figure 2B).

Specific observations have to be addressed when high levels of errors due to the switching of methylation status of $\mathrm{CpG}$ sites between the two groups have been simulated. In these scenarios, it was more difficult to single out a method that outperforms all competing approaches. However, when $\delta$ was as high as $10 \%$ (i.e., 1 in $10 \mathrm{CpGs}$ is misclassified as unmethylated or vice versa), we observed that ABBA was the best single method in 33 (46\%) of 72 simulated scenarios, whereas DSS and BSmooth performed as the best method in $16(22 \%)$ and $7(10 \%)$ cases, respectively, and, in other 10 cases, ABBA and DSS have comparable performance. The latter was more apparent when large probability differences between the two groups were simulated $(\Delta$ meth $=50$ or $70 \%)$.

We then explored whether nonhomogeneous, spatially correlated, read depth has an effect on the performance of ABBA. In order to capture spatially correlated read depth from real data, we sampled 5000 contiguous CpGs from WGBS data (generated in rat macrophages, see below and Materials and Methods for details), and then varied other parameters ( $r$ and $\Delta$ meth) using WGBSSuite as described above. In these "dataderived" simulated datasets, the read depth was correlated with the distance between CpGs (Figure S9A). The results of the benchmark using read depth taken from real data were very similar to those obtained using read depth simulated by means of a Poisson distribution (see Materials and Methods). Regardless of whether "data-derived" or "Poisson-simulated" read depth was used in our simulations, ABBA was the best performing method to recall DMRs (representative examples are reported in Figure S9B). While heterogeneous levels of read depth impact on the single base probability of methylation, the hierarchical model underlying ABBA borrows information across the sequence analyzed, it turns out that ABBA posterior estimates are less sensitive to different levels of the read depth.

Taken together, our simulation study shows that, while individual approaches can be very powerful in detecting DMRs under specific scenarios (notably, DSS with $r=1$ and BSmooth with $\Delta$ meth $=20 \%$ ), their performance can vary (and drop) significantly for different choices of the parameters tested in our simulations (at least within the parameter-space considered here). In contrast, we show that, on the whole, ABBA is the best performing method across a large number of parameters' combination tested, and accurately identifies DMRs in the large majority of simulated cases (Figure 2C). Specifically, ABBA's performance was the highest in the detection of biologically meaningful changes in DNA methylation ( $\Delta$ meth $\geq 30 \%$ ), and when little or no error due to random switching of methylation status of CpG sites between the two groups is present in the data.

DNA methylation is emerging as a major contributing factor in several human disorders (Zoghbi and Beaudet 2016), including important autoimmune diseases like systemic lupus erythematosus (SLE) (Wu et al. 2016). For instance, differential DNA methylation analysis in CD4+ T cells in lupus patients compared to normal healthy controls identified several genes with known involvement in autoimmunity (Jeffries et al. 2011). Here, to illustrate the practical utility of ABBA for differential methylation analysis in disease, we generated WGBS data in an established experimental rat model of crescentic glomerulonephritis (CRGN) (Aitman et al. 2006). In this model, we and others have previously shown that susceptibility to CRGN is mediated by macrophages (Behmoaras et al. 2008; Page et al. 2012); therefore, we assayed CpG methylation at single-nucleotide resolution by WGBS in primary macrophages derived from Wistar Kyoto (WKY) and Lewis (LEW) isogenic rats (two strains discordant for their predisposition to develop CRGN). We used ABBA to carry out genome-wide differential DNA methylation analysis in primary bone-marrow derived macrophages (BMDM) derived from the disease-prone rat strain (WKY, $r=4$ ) and control strain (LEW, $r=4$ ) - see Materials and Methods for additional details on WGBS data generation and processing. Briefly, in our ABBA analysis of the macrophage methylome, we used the following (default) settings: a minimum of five $\mathrm{CpG}$, and at least $33 \%$ difference in DNA methylation between the disease and control macrophages to identify DMRs. This choice was motivated and supported by data on the local topology of CpG sites in the methylome, showing that the vast majority of the CpG clusters are in the range of 1-11 CpGs (Lövkvist et al. 2016), and to increase true positive rate in our DM analysis, following previous assessment and recommendations for methylation analysis using WGBS data (Ziller et al. 2015).

Using an FDR cutoff of 5\%, ABBA identified 1004 DMRs genome-wide, with $1.07 \%$ falling within an annotated CpGI, and $6.78 \%$ within an annotated CpGS (Figure 3A). For comparative purposes, we also used DSS (since this method performed very similarly to ABBA in several simulated cases, Figure 2) to identify DMRs genome-wide, which resulted in only 207 regions with significant differential methylation (uncorrected $P$-value threshold $=10^{-3}$, using the default parameters of DSS). Of the 1004 DMRs identified by ABBA, 427 overlapped with annotated genes (Table S3), and there was a significant enrichment for DMRs occurring within $1 \mathrm{~kb}$ of the gene boundaries $(P$-value $<0.001)$, within exons ( $P$ value $<0.05)$, and within introns $(P$-value $<0.05$; Figure 3B). The genes that are within $1 \mathrm{~kb}$ of a DMR were enriched for pathways relevant to the pathophysiology of CRGN, including MAPK signaling (Ryan et al. 2011), Phosphatidylinositol signaling (Wu et al. 2014) and Fc gamma R-mediated phagocytosis (Page et al. 2012) (Figure 3C). For comparison, 
the 207 DMRs identified by DSS overlapped with 45 genes (Table S4), which were enriched only for RNA degradation and metabolic pathways. The analysis of real WGBS data by DSS highlighted how the choice of parameters (in this case related to the size of the moving average window in the smoothing procedure) can affect the results. Since the window size in DSS is a user-defined parameter, we performed the analysis with DSS using three different windows (50, 100 , and $1000 \mathrm{bp}$ ) in addition to the default window size of $500 \mathrm{bp}$. Each of the four window sizes identified a different number of DMRs, which overlap with different genes (Figure S10A), and have varying distributions of DMR lengths (Figure S10, B-E). The genes identified by DSS when a window of $50 \mathrm{bp}$ is used showed no significant enrichment for pathways, while the results obtained for $100 \mathrm{bp}$ and $1000 \mathrm{bp}$ windows showed a significant enrichment for RNA degradation. These analyses highlight how the arbitrary choice of parameters related to the degree of smoothing can affect greatly the results of a genome-wide DM analysis as well as the downstream annotation of the genes overlapping with DMRs. In contrast, ABBA automatically adapts to different correlation structures in DNA methylation levels across the genome without requiring any user-defined parameters related to the smoothing procedure.

As DNA methylation can affect gene expression by interfering with transcription factor (TF) binding, we performed a TFBS analysis of the DMRs (Figure 3D). This revealed significant enrichment for several TFs, including the ETS transcription factors family and a number of proteins that make the AP-1 TF complex (JUNB, FOS, JUN, and JUND), which have been previously linked with CRGN (Raffetseder et al. 2004; Behmoaras et al. 2008). To further investigate the potential effect of the changes in DNA methylation identified by ABBA, we carried out differential expression (DE) analysis in macrophages from WKY and LEW rats by RNA-seq (see Materials and Methods for details). The list of DE genes $[n=910$, Benjamini-Hochberg (BH)-corrected $P$-value $<0.05]$ was crosschecked with the genes impacted by DMRs (above), identifying 48 genes with both significant differential methylation and DE (Table S5). We observed the "textbook" model describing DNA methylation regulating transcription via the promoter region (i.e., hypermethylation in the promoter associated with transcriptional repression, see below), as well as widespread methylation changes in the genes body and 3'UTR associated with both gene repression and activation. The genes with concordant promoter hypermethylation and transcriptional repression, Ifitm3, Ydjc, and Cd300Ig were investigated in more detail since the gene's promoter is a key regulatory region where the effect of DNA methylation is more clearly understood. We found the biggest change in mRNA expression was in interferon induced transmembrane protein 3 (Ifitm3), with mRNA from this gene being almost undetected in unstimulated WKY macrophages (Figure $3 \mathrm{E}$ ). This observation is consistent with the differential methylation status of the promoter of Ifitm3, where the WKY rats had higher levels of methylation than the LEW rats
(Figure 3F). To further support the identification of differential methylation at the Ifitm3 gene, we checked whether other methods identified the same DMR. While MethylSig failed to identify significant DMR and BSmooth identified a large and unspecific genomic area as differentially methylated, DSS provides highly consistent results with ABBA, identifying differential methylation at the same region at the Ifitm3 gene promoter (Figure S11).

We have previously shown that the JunD (AP-1) transcription factor is a major determinant of CRGN in WKY rats (Behmoaras et al. 2008), and others have shown that AP-1 is methylation sensitive (Ogawa et al. 2014). Therefore we scanned the DMR (spanning $600 \mathrm{bp}$ ) for canonical JunD binding site motifs, and identified four putative regions in the promoter region of Ifitm3 (Figure 3G). In addition, we reanalyzed ChIP-seq data for a JunD transcription factor in BMDM derived from WKY and a congenic strain from LEW (see Materials and Methods for details). This analysis identified significant differences in JunD binding between WKY and the LEW-congenic strain that overlapped with two of the four TFBS identified at the Ifitm3 promoter (Figure $3 G)$. The combined evidence provided by our ABBA analysis and RNA-seq/ChIP-seq data therefore suggests that the effect of DNA methylation of the Ifitm3 gene promoter in WKY rats (prone to develop CRGN) may be restricting the binding of transcription factors such as JunD, and, as a consequence, the gene is almost not expressed $<1$ tags per million in unstimulated macrophages of WKY rats.

\section{Discussion}

As the cost of genome sequencing technologies continues to drop, it will soon become commonplace to perform comprehensive methylome analyses, using WGBS or other highthroughput techniques that allow the unbiased genome-wide quantification of DNA methylation at a single base-pair resolution. However, high-resolution data generation is only the first step toward the identification of genomic loci, and eventually genes with altered methylation levels associated with a given disease, phenotype, or developmental stage. The number of DNA methylation datasets available in the public domain is expected to grow; therefore, it becomes necessary to provide the scientific community with analytical tools for a reliable and reproducible identification of differential methylation, and facilitate large epigenome-mapping projects and epigenome-wide association studies (Bock 2012).

Beyond statistical power considerations specifically related to sample size (Rakyan et al. 2011) or interpretability of epigenome-wide association studies (Birney et al. 2016), our ability to identify accurately changes in DNA methylation localized to specific genomic loci (genes) is also influenced by multiple factors inherently correlated to data quality. These include the within-group heterogeneity, the level of noise, the presence of known genetic covariates (Zhang et al. 2015), and nongenetic confounding factors (e.g., batch effects), as well as features such as sequencing depth (Ziller et al. 2015), 
or errors due incomplete bisulfite conversion (Genereux et al. 2008). Therefore, any analytical tool that can account for all these factors will reduce the number of false positives, maximizing the sensitivity, and calling the regions of interest (i.e., differentially methylated) as accurately as possible. With this in mind, we designed a differential methylation analysis tool (ABBA) that is robust to different experimental and technical variables (see Figure 2), and that adapts automatically to the varying genomic context and local topology of CpG sites affecting methylation levels. In particular, the automatic adaptation to different correlation structures in $\mathrm{CpG}$ methylation levels (without requiring user-defined parameters about the degree of smoothing), as well as the ability of modeling its decay as a function of the genomic distances between CpGs allow ABBA to adapt routinely to methylation changes that occur with different scales and at nonuniform rates across the genome. The importance of the genomic context in the methylome, and the local topology of CpG sites have been recently investigated, showing, among other features, that methylation at small CpG clusters is more likely to induce stable changes in DNA methylation (Lövkvist et al. 2016).

From a user's perspective, ABBA treats WGBS-seq data in a general way with no specification of parameters related to the level of data smoothing (such as window size or kernel bandwidth), thus allowing for a great deal of automation. This also facilitates the WGBS analysis when the values of the parameter settings (that may greatly affect the accuracy of DM identification) are not known. Our fully Bayesian approach can also be easily modified to include covariates and nongenetic confounding factors through random effects, beyond the replicates level. It also allows the specification of covariates that are informative about the methylation profiles by adding prior biological information to the linear predictor $\mu_{i g}$ in Equation (6). While these alterations can be made in our model with a simple modification of the code, and with negligible further computational costs, nonparametric smoothing techniques [spline- (Hansen et al. 2012), kernel- (Hebestreit et al. 2013), and moving average-based smoothing (Feng et al. 2014)] do not possess the same straightforward flexibility, nor do alternative approaches based on Hidden Markov Models (Kuan and Chiang 2012; Sun and Yu 2016; Yu and Sun 2016a).

Our extensive simulation studies (Figure 2) and differential DNA methylation analysis in glomerulonephritis (Figure 3) showed that ABBA is a powerful approach for the identification of DMRs from WGBS single-base pair resolution methylation data. While individual methods such as BSmooth (Hansen et al. 2012) or DSS (Feng et al. 2014; Wu et al. 2015) showed a very good power to detect DMRs under specific scenarios and conditions, ABBA retained a high degree of robustness of the results with respect to a wider range of factors (parameters) affecting WGBS data integrity and quality, including sequencing coverage, number of replicates, or different noise structures. This is particularly appealing in cases when considerable efforts have been expended toward generation of large-scale WGBS data from heterogeneous systems, e.g., the ENCODE project (Bernstein et al.
2012), and data quality can vary across experimental conditions and laboratories. As proof of concept of ABBA's application to real data analysis, we used an established experimental model system of glomerulonephritis (Aitman et al. 2006) to identify changes in DNA methylation associated with disease. In this, we employed ABBA to analyze $\sim 15$ million $\mathrm{CpG}$ sites genome-wide in primary bone-marrow derived macrophages derived from WKY and LEW rats, and identified $>1000$ significant DMRs at 5\% FDR level. A comparative analysis using DSS (the most competitive approach from our simulation study) did not provide the same level of biological insight both in terms of significant pathway enrichments, and in robustly identifying DMRs across user-defined parameters. To highlight this point, we showed how the results of DSS were greatly affected by the choice of the window size.

Furthermore, we have shown how integrating the DMR results provided by ABBA with other "omics" data (RNA-seq and ChIP-seq generated in the same experimental system), enabled us to generate new hypotheses for the mechanism underpinning the disease, revealing a candidate gene (Ifitm3) for the susceptibility to glomerulonephritis. These findings on Ifitm3 in rat glomerulonephritis merit further discussion. Ifitim 3 has a known role in viral resistance, a central part of innate immunity, and is inducible by both interferon (IFN) types I and II (Everitt et al. 2012). Notably, type II IFN signaling has been implicated in the pathogenesis of nephrotoxic nephritis, and other "planted" antigen models of CRGN (Kitching et al. 2004), although DNA methylation has not previously been examined in this context. With regards to type I IFN, recent genome-wide DNA methylation analysis of T-cells, B-cells, and monocytes has shown that patients with SLE, a frequent autoimmune cause of CRGN, have severe hypomethylation near to genes involved in type I IFN signaling (Absher et al. 2013). In addition, DNA methylation alterations in IFN-related genes, including Ifitm3, have been previously observed and proposed to contribute to the pathogenesis of other autoimmune diseases such as primary Sjögren's syndrome (Gottenberg et al. 2006). Regarding the role of Ifitm 3 , it has been shown to directly interact in vivo and in vitro, with osteopontin, a matricellular protein whose transcription is mediated by the AP-1 TF family (El-Tanani et al. 2010). Furthermore, osteopontin has been also previously associated with SLE (Rullo et al. 2013), and ANCAassociated vasculitis (Lorenzen et al. 2010) —another frequent cause of CRGN. Therefore, our ABBA analysis of WGBS data in primary macrophages from a rat model of CRGN allowed us to propose an AP-1-mediated role for Ifitm3 in glomerulonephritis. While a role for IFN-signaling genes in autoimmune disease has been previously suggested, our findings on methylation alteration of the Ifitm 3 gene associated with glomerulonephritis in the rat might suggest future directions for the study of the pathogenesis, and to develop treatments of CRGN.

In a wider context, the role of methylation is dependent on the location with respect to the gene body and regulation functions. Methylation in a CpGI-depleted promoter, such as the promoter region of Ifitm3 gene [according to UCSC genome browser (RN4)], is associated with repression that 
maybe due to interference with transcription factor binding. Conversely, methylation in the gene body is positively associated with active transcription as methylation can be caused by transcriptional elongation (Schübeler 2015). Methylation within a gene body can also act as an insulator for repetitive and transposable elements or distal intronic enhancers, on which the methylation would have no regulatory effect on the gene in which it resides (Jones 2012). Given the complexity of these regulatory functions of methylation, the ability of our approach to accurately identify changes in DNA methylation that are localized to specific regions is likely to facilitate our understanding of the complex relationships between methylation and gene regulation. As exemplified by our integrative analysis of the Ifitm 3 locus, we anticipate that the ABBA results for differential DNA methylation should be integrated with additional transcriptional and epigenetic data in order to better define hypotheses on specific regulatory mechanisms.

In summary, we show how ABBA provides a flexible and user-friendly automatic framework for the identification of differential methylation that is robust across a wide range of experimental parameters-an approach that we have also applied to identify changes in macrophage DNA methylation in glomerulonephritis.

\section{Acknowledgments}

The authors are thankful to the two anonymous referees whose meticulous attention to their refereeing task has resulted in substantial improvements in the presentation. This research was funded by Engineering and Physical Sciences Research Council Grant EP/K030760/1 (L.B.), The Alan Turing Institute under the Engineering and Physical Sciences Research Council grant EP/N510129/1 (L.B. and P.D.), Royal Society IE110977 (L.B. and P.D.), European Union (European Social Fund), Greek national funds through the Operational Program "Education and Lifelong Learning" of the National Strategic Reference Framework, project ARISTEIA (P.D.), Duke-National University of Singapore Medical School, and Singapore Ministry of Health (O.J. L.R. and E.P.), a Medical Research Council Chain-Florey fellowship (T.O.), the Medical Research Council (MR/ M004716/1 to J.B. and E.P.), and by Kidney Research UK-RP9/2013 (J.B.). The funders had no role in study design, data collection and analysis, decision to publish, or preparation of the manuscript.

Author contributions: L.B. and E.P. initiated, directed, and supervised the project. P.D. and L.B. conceived the statistical model and the computational approach. P.D., E.V., and L.B. wrote the initial algorithm that was further developed by O.J.L.R. and L.B. to the presented approach. T.O. and E.P. generated WGBS data in the rat. S.R.L., O.J.L.R., and E.P. carried out analysis of WGBS and RNA-seq data in the rat and interpreted the results. N.H. and P.K.S. carried out ChIP-seq and TFBS analyses. J.B. provided RNA-seq and
ChIP-seq data in the rat. O.J.L.R., L.B., and E.P. wrote the manuscript with input from all authors. All of the authors read and approved the final manuscript.

\section{Literature Cited}

Absher, D. M., X. Li, L. L. Waite, A. Gibson, K. Roberts et al., 2013 Genome-wide DNA methylation analysis of systemic lupus erythematosus reveals persistent hypomethylation of interferon genes and compositional changes to CD4+ T-cell populations. PLoS Genet. 9: e1003678.

Äijö, T., Y. Huang, H. Mannerström, L. Chavez, A. Tsagaratou et al., 2016 A probabilistic generative model for quantification of DNA modifications enables analysis of demethylation pathways. Genome Biol. 17: 49.

Aitman, T. J., R. Dong, T. J. Vyse, P. J. Norsworthy, M. D. Johnson et al., 2006 Copy number polymorphism in Fcgr3 predisposes to glomerulonephritis in rats and humans. Nature 439: 851855.

Akalin, A., M. Kormaksson, S. Li, F. E. Garrett-Bakelman, M. E. Figueroa et al., 2012 methylKit: a comprehensive R package for the analysis of genome-wide DNA methylation profiles. Genome Biol. 13: R87.

Anders, S., A. Reyes, and W. Huber, 2012 Detecting differential usage of exons from RNA-seq data. Genome Res. 22: 20082017.

Anders, S., P. T. Pyl, and W. Huber, 2015 HTSeq-a Python framework to work with high-throughput sequencing data. Bioinformatics 31: 166-169.

Behmoaras, J., G. Bhangal, J. Smith, K. McDonald, B. Mutch et al., 2008 Jund is a determinant of macrophage activation and is associated with glomerulonephritis susceptibility. Nat. Genet. 40: 553-559.

Bell, J. T., A. A. Pai, J. K. Pickrell, D. J. Gaffney, R. Pique-Regi et al., 2011 DNA methylation patterns associate with genetic and gene expression variation in HapMap cell lines. Genome Biol. 12: R10.

Bernstein, B. E., E. Birney, I. Dunham, E. D. Green, C. Gunter et al., 2012 An integrated encyclopedia of DNA elements in the human genome. Nature 489: 57-74.

Birney, E., G. D. Smith, and J. M. Greally, 2016 Epigenome-wide association studies and the interpretation of disease -omics. PLoS Genet. 12: e1006105.

Bock, C., 2012 Analysing and interpreting DNA methylation data. Nat. Rev. Genet. 13: 705-719.

Broët, P., A. Lewin, S. Richardson, C. Dalmasso, and H. Magdelenat, 2004 A mixture model-based strategy for selecting sets of genes in multiclass response microarray experiments. Bioinformatics 20: 2562-2571.

Chen, Z.-X., and A. D. Riggs, 2011 DNA methylation and demethylation in mammals. J. Biol. Chem. 286: 18347-18353.

Deaton, A. M., and A. Bird, 2011 CpG islands and the regulation of transcription. Genes Dev. 25: 1010-1022.

Dempster, A. P. A., N. M. N. Laird, and D. D. B. Rubin, 1977 Maximum likelihood from incomplete data via the EM algorithm. J. R. Stat. Soc. B 39: 1-38.

Doucet, A., N. de Freitas, and N. Gordon, 2013 Sequential Monte Carlo Methods in Practice. Springer Science \& Business Media, Berlin.

Efron, B., 2008 Simultaneous inference: when should hypothesis testing problems be combined? Ann. Appl. Stat. 2: 197-223.

El-Tanani, M. K., D. Jin, F. C. Campbell, and P. G. Johnston, 2010 Interferon-induced transmembrane 3 binds osteopontin in vitro: expressed in vivo IFITM3 reduced OPN expression. Oncogene 29: 752-762. 
Everitt, A. R., S. Clare, T. Pertel, S. P. John, R. S. Wash et al., 2012 IFITM3 restricts the morbidity and mortality associated with influenza. Nature 484: 519-523.

Feng, H., K. N. Conneely, and H. Wu, 2014 A Bayesian hierarchical model to detect differentially methylated loci from single nucleotide resolution sequencing data. Nucleic Acids Res. 42: e69.

Genereux, D. P., W. C. Johnson, A. F. Burden, R. Stöger, and C. D. Laird, 2008 Errors in the bisulfite conversion of DNA: modulating inappropriate- and failed-conversion frequencies. Nucleic Acids Res. 36: e150.

Gilks, W. R., S. Richardson, and D. J. Spiegelhalter, 1996 Markov Chain Monte Carlo in Practice. Chapman and Hall, London.

Gottenberg, J.-E., N. Cagnard, C. Lucchesi, F. Letourneur, S. Mistou et al., 2006 Activation of IFN pathways and plasmacytoid dendritic cell recruitment in target organs of primary Sjögren's syndrome. Proc. Natl. Acad. Sci. USA 103: 2770-2775.

Gutierrez-Arcelus, M., T. Lappalainen, S. B. Montgomery, A. Buil, H. Ongen et al., 2013 Passive and active DNA methylation and the interplay with genetic variation in gene regulation. eLife 2: e00523.

Gutierrez-Arcelus, M., H. Ongen, T. Lappalainen, S. B. Montgomery, A. Buil et al., 2015 Tissue-specific effects of genetic and epigenetic variation on gene regulation and splicing. PLoS Genet. 11: e1004958.

Hansen, K. D., B. Langmead, and R. A. Irizarry, 2012 BSmooth: from whole genome bisulfite sequencing reads to differentially methylated regions. Genome Biol. 13: R83.

Harris, R. A., T. Wang, C. Coarfa, R. P. Nagarajan, C. Hong et al., 2010 Comparison of sequencing-based methods to profile DNA methylation and identification of monoallelic epigenetic modifications. Nat. Biotechnol. 28: 1097-1105.

Hebestreit, K., M. Dugas, and H.-U. Klein, 2013 Detection of significantly differentially methylated regions in targeted bisulfite sequencing data. Bioinformatics 29: 1647-1653.

Heinz, S., C. Benner, N. Spann, E. Bertolino, Y. C. Lin et al., 2010 Simple combinations of lineage-determining transcription factors prime cis-regulatory elements required for macrophage and B cell identities. Mol. Cell 38: 576-589.

Hull, R. P., P. K. Srivastava, Z. D'Souza, S. S. Atanur, F. MechtaGrigoriou et al., 2013 Combined ChIP-Seq and transcriptome analysis identifies AP-1/JunD as a primary regulator of oxidative stress and IL-1 $\beta$ synthesis in macrophages. BMC Genomics 14: 92.

Jeffries, M. A., M. Dozmorov, Y. Tang, J. T. Merrill, J. D. Wren et al., 2011 Genome-wide DNA methylation patterns in CD4+ $\mathrm{T}$ cells from patients with systemic lupus erythematosus. Epigenetics 6: 593-601.

Johnson, M. D., M. Mueller, L. Game, and T. J. Aitman, 2012 Single nucleotide analysis of cytosine methylation by whole-genome shotgun bisulfite sequencing, pp. 21.23 in Current Protocols in Molecular Biology. John Wiley \& Sons, Inc., Hoboken, NJ.

Johnson, M. D., M. Mueller, M. Adamowicz-Brice, M. J. Collins, P. Gellert et al., 2014 Genetic analysis of the cardiac methylome at single nucleotide resolution in a model of human cardiovascular disease. PLoS Genet. 10: e1004813.

Jones, P. A., 2012 Functions of DNA methylation: islands, start sites, gene bodies and beyond. Nat. Rev. Genet. 13: 484-492.

Jühling, F., H. Kretzmer, S. H. Bernhart, C. Otto, P. F. Stadler et al., 2016 metilene: fast and sensitive calling of differentially methylated regions from bisulfite sequencing data. Genome Res. 26: 256-262.

Kitching, A. R., A. L. Turner, T. Semple, M. Li, K. L. Edgtton et al., 2004 Experimental autoimmune anti-glomerular basement membrane glomerulonephritis: a protective role for IFNgamma. J. Am. Soc. Nephrol. 15: 1764-1774.
Kuan, P. F., and D. Y. Chiang, 2012 Integrating prior knowledge in multiple testing under dependence with applications to detecting differential DNA methylation. Biometrics 68: 774783.

Lea, A. J., J. Tung, and X. Zhou, 2015 A flexible, efficient binomial mixed model for identifying differential DNA methylation in bisulfite sequencing data. PLOS Genet. 11: e1005650.

Li, H., and R. Durbin, 2009 Fast and accurate short read alignment with Burrows-Wheeler transform. Bioinformatics 25: $1754-1760$.

Lorenzen, J., S. Lovric, R. Krämer, H. Haller, and M. Haubitz, 2010 Osteopontin in antineutrophil cytoplasmic autoantibody-associated vasculitis: relation to disease activity, organ manifestation and immunosuppressive therapy. Ann. Rheum. Dis. 69: 1169-1171.

Love, M. I., W. Huber, and S. Anders, 2014 Moderated estimation of fold change and dispersion for RNA-seq data with DESeq2. Genome Biol. 15: 550.

Lövkvist, C., I. B. Dodd, K. Sneppen, and J. O. Haerter, 2016 DNA methylation in human epigenomes depends on local topology of CpG sites. Nucleic Acids Res. 44: 5123-5132.

Ma, H., A. I. Bandos, H. E. Rockette, and D. Gur, 2013 On use of partial area under the ROC curve for evaluation of diagnostic performance. Stat. Med. 32: 3449-3458.

Mathelier, A., X. Zhao, A. W. Zhang, F. Parcy, R. Worsley-Hunt et al., 2014 JASPAR 2014: an extensively expanded and updated open-access database of transcription factor binding profiles. Nucleic Acids Res. 42: D142-D147.

Ogawa, C., Y. Tone, M. Tsuda, C. Peter, H. Waldmann et al., 2014 TGF- $\beta$-mediated Foxp3 gene expression is cooperatively regulated by Stat5, Creb, and AP-1 through CNS2. J. Immunol. 192: 475-483.

Page, T. H., Z. D'Souza, S. Nakanishi, T. Serikawa, C. D. Pusey et al., 2012 Role of novel rat-specific Fc receptor in macrophage activation associated with crescentic glomerulonephritis. J. Biol. Chem. 287: 5710-5719.

Park, Y., M. E. Figueroa, L. S. Rozek, and M. A. Sartor, 2014 MethylSig: a whole genome DNA methylation analysis pipeline. Bioinformatics 30: 2414-2422.

Rackham, O. J. L., P. Dellaportas, E. Petretto, and L. Bottolo, 2015 WGBSSuite: simulating whole-genome bisulphite sequencing data and benchmarking differential DNA methylation analysis tools. Bioinformatics 31: 2371-2373.

Raffetseder, U., N. Wernert, T. Ostendorf, C. van Roeyen, T. Rauen et al., 2004 Mesangial cell expression of proto-oncogene Ets-1 during progression of mesangioproliferative glomerulonephritis. Kidney Int. 66: 622-632.

Rakyan, V. K., T. A. Down, D. J. Balding, and S. Beck, 2011 Epigenome-wide association studies for common human diseases. Nat. Rev. Genet. 12: 529-541.

Robinson, M. D., A. Kahraman, C. W. Law, H. Lindsay, M. Nowicka et al., 2014 Statistical methods for detecting differentially methylated loci and regions. Front. Genet. 5: 324.

Rotival, M., J.-H. Ko, P. K. Srivastava, A. Kerloc'h, A. Montoya et al., 2015 Integrating phosphoproteome and transcriptome reveals new determinants of macrophage multinucleation. Mol. Cell. Proteomics 14: 484-498.

Rue, H., and S. Martino, 2007 Approximate Bayesian inference for hierarchical Gaussian Markov random field models. J. Stat. Plan. Inference 137: 3177-3192.

Rue, H., S. Martino, and N. Chopin, 2009 Approximate Bayesian inference for latent Gaussian models by using integrated nested Laplace approximations. J. R. Stat. Soc. B 71: 319-392.

Rullo, O. J., J. M. P. Woo, M. F. Parsa, A. D. C. Hoftman, P. Maranian et al., 2013 Plasma levels of osteopontin identify patients at risk for organ damage in systemic lupus erythematosus. Arthritis Res. Ther. 15: R18. 
Ryan, J., F. Y. Ma, J. Kanellis, M. Delgado, K. Blease et al., 2011 Spleen tyrosine kinase promotes acute neutrophilmediated glomerular injury via activation of JNK and p38 MAPK in rat nephrotoxic serum nephritis. Lab. Invest. 91: 1727-1738.

Schübeler, D., 2015 Function and information content of DNA methylation. Nature 517: 321-326.

Srivastava, P. K., R. P. Hull, J. Behmoaras, E. Petretto, and T. J. Aitman, 2013 D/AP1 regulatory network analysis during macrophage activation in a rat model of crescentic glomerulonephritis. BMC Syst. Biol. 7: 93.

Sun, D., Y. Xi, B. Rodriguez, H. J. Park, P. Tong et al., 2014 MOABS: model based analysis of bisulfite sequencing data. Genome Biol. 15: R38.

Sun, S., and X. Yu, 2016 HMM-Fisher: identifying differential methylation using a hidden Markov model and Fisher's exacttest. Stat. Appl. Genet. Mol. Biol. 15: 55-67.

Sun, W., and T. Tony Cai, 2009 Large-scale multiple testing under dependence. J. R. Stat. Soc. Ser. B. Stat. Methodol. 71: 393424.

Tierney, L., and J. B. Kadane, 1986 Accurate approximations for posterior moments and marginal densities. J. Am. Stat. Assoc. 81: 82-86.

Wang, J., D. Duncan, Z. Shi, and B. Zhang, 2013 WEB-based GEne SeT AnaLysis Toolkit (WebGestalt): update 2013. Nucleic Acids Res. 41: W77-W83.

Wu, H., T. Xu, H. Feng, L. Chen, B. Li et al., 2015 Detection of differentially methylated regions from whole-genome bisulfite sequencing data without replicates. Nucleic Acids Res. 43: e141.
Wu, H., M. Zhao, L. Tan, and Q. Lu, 2016 The key culprit in the pathogenesis of systemic lupus erythematosus: aberrant DNA methylation. Autoimmun. Rev. 15: 684-689.

Wu, T., Y. Ye, S.-Y. Min, J. Zhu, E. Khobahy et al., 2014 Prevention of murine lupus nephritis by targeting multiple signaling axes and oxidative stress using a synthetic triterpenoid. Arthritis Rheumatol. 66: 3129-3139.

Yu, X., and S. Sun, 2016a HMM-DM: identifying differentially methylated regions using a hidden Markov model. Stat. Appl. Genet. Mol. Biol. 15: 69-81.

Yu, X., and S. Sun, 2016b Comparing five statistical methods of differential methylation identification using bisulfite sequencing data. Stat. Appl. Genet. Mol. Biol. 15: 173-191.

Zhang, W., T. D. Spector, P. Deloukas, J. T. Bell, and B. E. Engelhardt, 2015 Predicting genome-wide DNA methylation using methylation marks, genomic position, and DNA regulatory elements. Genome Biol. 16: 14.

Ziller, M. J., F. Müller, J. Liao, Y. Zhang, H. Gu et al., 2011 Genomic distribution and inter-sample variation of non-CpG methylation across human cell types. PLoS Genet. 7: e1002389.

Ziller, M. J., K. D. Hansen, A. Meissner, and M. J. Aryee, 2015 Coverage recommendations for methylation analysis by whole-genome bisulfite sequencing. Nat. Methods 12: 230-232.

Zoghbi, H. Y., and A. L. Beaudet, 2016 Epigenetics and human disease. Cold Spring Harb. Perspect. Biol. 8: a019497.

Communicating editor: A. Moses 


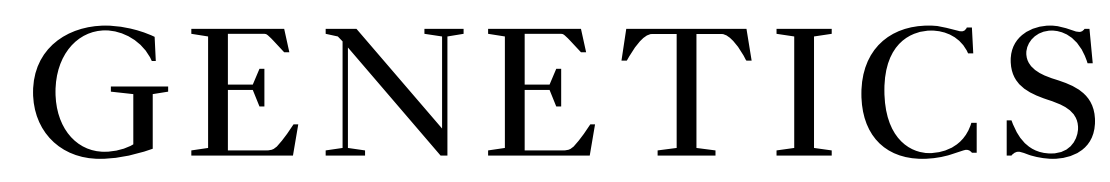

Supporting Information www.genetics.org/lookup/suppl/doi:10.1534/genetics.116.195008/-/DC1

\section{A Bayesian Approach for Analysis of Whole-Genome Bi- sulfite Sequencing Data Identifies Disease-Associated Changes in DNA Methylation}

Owen J. L. Rackham, Sarah R. Langley, Thomas Oates, Eleni Vradi, Nathan Harmston, Prashant K. Srivastava, Jacques Behmoaras, Petros Dellaportas, Leonardo Bottolo and Enrico Petretto 